\title{
Mixture toxicity analysis in zebrafish embryo: a time and concentration resolved study on mixture effect predictivity
}

\author{
Gianina Jakobs ${ }^{1,2^{*}}$ (D) Janet Krüger ${ }^{1}$, Andreas Schüttler ${ }^{1} \mathbb{B}$, Rolf Altenburger ${ }^{1,2}$ and Wibke Busch ${ }^{1}$ (1)
}

\begin{abstract}
Background: Humans and wildlife are continuously exposed to chemical mixtures. These mixtures vary in composition but typically contain hundreds of micropollutants at low concentrations. As it is not feasible to measure the toxicity of all possibly occurring mixtures, there is a need to predict mixture toxicity. Two models, Concentration Addition (CA) and Independent Action (IA), have been applied to estimate mixture toxicity. Here, we compared measured with predicted toxicity of nine mixtures designed from 15 environmentally relevant substances in zebrafish embryos to investigate the usability of these models for predicting phenotypic effects in a whole organism short term acute assay.

Results: In total, we compared 177 toxicity values derived from 31 exposure scenarios with their predicted counterparts. Our results show that mixture toxicity was either correctly estimated (86\%) by the prediction window, the concentration-effect space that is spanned between both models, or was underestimated with both models (14\%). The CA model correctly predicted the measured mixture toxicity in $100 \%$ of cases when a prediction deviation factor of 2.5 was allowed. However, prediction accuracy of mixture toxicity prediction was dependent on exposure duration and mixture potency. The CA model showed highest prediction quality for long-term exposure with highly potent mixtures, respectively, whereas IA proved to be more accurate for short-term exposure with less potent mixtures. Obtained mixture concentration-response curves were steep and indicated the occurrence of remarkable combined effects as mixture constituents were applied at concentrations below their respective individual effect threshold in $90 \%$ of all investigated cases.

Conclusions: Experimental factors, such as exposure duration or mixture potency, influence the prediction accuracy of both inspected models. The CA model showed highest prediction accuracy even for a set of diverse mixtures and various exposure conditions. However, the prediction window served as the most robust predicator to estimate mixture toxicity. Overall, our results demonstrate the importance of considering mixture toxicity in risk assessment schemes and give guidance for future experiment design regarding mixture toxicity investigations.
\end{abstract}

Keywords: Zebrafish embryo acute toxicity assay, Mixture toxicity, Prediction window, Concentration Addition (CA), Independent Action (IA), 'Something from "nothing"', Micropollutants

*Correspondence: gianina.jakobs@ufz.de

${ }^{1}$ Department of Bioanalytical Ecotoxicology, UFZ-Helmholtz Centre

for Environmental Research, Permoserstr. 15, 04318 Leipzig, Germany

Full list of author information is available at the end of the article

\section{Background}

Over the course of the last century, modernization, globalization, and population growth caused a tremendous increase in the amount of synthesized chemicals. Most of them are used for agricultural, industrial, domestic or medical purposes on a regular basis and are considered essential to modern life [1]. These chemicals end up 
in the environment through spills on land, emissions to air, and discharges to water. Consequently, humans and wildlife are continuously exposed to complex chemical mixtures rather than individual entities. These mixtures vary in composition but typically contain hundreds of micropollutants at low concentrations [2,3]. Exposure to low-concentrated chemical mixtures causes experimentally proven toxicity and scientific evidence of elevated toxicity in comparison to single substance exposures is urgently requesting for mixture toxicity assessment in water quality regulation [4-8]. However, current regulation is still based on individual chemical toxicity assessment. As it is not feasible to measure the toxicity of all possibly occurring mixtures, there is a critical need to develop approaches to reliably predict mixture toxicity. Two models, the concept of Concentration Addition (CA) and Independent Action (IA), have been shown and applied to predict mixture toxicity from known effects of the mixture components. CA implies that the effect induced by a mixture containing chemicals with similar modes of action (MoAs), hence addressing the same molecular target site, remains constant even if one chemical is replaced by an equal fraction of an equally effective concentration of another chemical. The CA concept also assumes that every toxicant in any concentration contributes, in proportion to its toxic unit, to the overall combined effect of a mixture $[9,10]$. In contrast, IA predicts the combined effect by multiplying the relative effect of independently acting mixture components, hence chemicals that exert their toxicity through an interaction with different molecular target sites [11]. The formulation of the IA concept indicates that only mixture constituents that are present in concentrations higher than their individual effect threshold (i.e., lowest observed effect concentrations) are able to contribute to the combined effect of a mixture $[10,11]$. Comparing both models, CA usually predicts lower mixture effect concentrations when regarding ecotoxicological studies [12-14]. This is especially the case when the prediction is based on chemicals that induce steep concentration-response curves (CRCs) [15]. Steep CRCs are particularly observed when integrated endpoints, such as mortality, growth, or quantity of malformations are considered [15, 16]. As mentioned before, the CA model implies that a significant mixture effect is possible to be detected even if mixture constituents are present in concentrations that fail to evoke individual toxicity. This phenomenon is known as the principle 'something from "nothing" [17, 18]. Several laboratory and field studies [10-16] confirmed this concept. So far, CA seems to reliably predict the combined effect caused by similarly acting components in multiple test systems, such as the freshwater algae Scenedesmus vacuolatus [19-21], luminescent bacteria Vibrio fischeri
[21], Daphnia magna [22, 23], guppy [24], and fathead minnow [25]. The toxicity induced by mixtures of dissimilarly acting substances, on the other hand, was only predictable with the IA model in rather simple organisms, e.g., Scenedesmus vacuolatus [13], and Vibrio fischeri [26]. In vertebrates, however, the IA model did not adequately estimate the mixture toxicity of dissimilarly acting components as expected [e.g., 19]. Hence, it is still not clarified whether the toxicity of a mixture of dissimilarly acting components is predictable with the IA model in complex organisms (e.g., fish) and the question whether chemicals with different MoAs are able to exert their toxicity independently is not answered yet. Furthermore, it remains unclear if the accuracy of mixture toxicity prediction models is dependent on the observed phenotype (e.g., lethal, sublethal, or teratogenic effects), mixture potency (e.g., $\mathrm{LC} / \mathrm{EC}_{10,50,90}$ ), or applied exposure duration.

To address these questions, we performed a time, concentration, and effect resolved mixture toxicity analysis in zebrafish embryo (ZFE, Danio rerio) and determined how the mixture potency and composition, exposure duration, and considered phenotype influence the predictability of mixture toxicity with the CA and IA model. Therefore, we analyzed the mixture toxicity of nine mixtures that were composed of 15 environmentally relevant substances. Hence, mixtures were comparable but varied in composition and chemical concentration. Some mixtures contained only components presumed to act similarly or only dissimilarly, whereas other mixtures contained a blend of both. The combination of the applied mixture design and selection of the ZFE as model organism provided the opportunity to examine whether the CA and IA model correctly estimate the toxicity of respective mixtures in a complex organism. Furthermore, the ZFE undergoes several developmental stages from zygote to larvae within the first $96 \mathrm{~h}$ post fertilization (hpf) [27] and is, therefore, an excellent model organism to investigate the impact of exposure period on mixture toxicity predictability. Additionally, transparency of ZFE offers the opportunity to microscopically detect multiple lethal, sublethal, and teratogenic effects. Overall, we measured the toxicity of 31 different exposure scenarios and compared 177 measured toxicity values with their counterparts predicted with the CA and IA model. We calculated the distance between measured and predicted toxicity, the prediction deviation ratio, and determined the prediction window which is defined as the concentration-effect space that is spanned between both models. We further inspected the general appearance of mixture concentration-response curves and analyzed the ability of a mixture to induce a combined toxic effect, particularly whether the principle 'something from "nothing" applies for the analyzed mixtures. With this approach, 
we (1) provide a comprehensive, time and concentration resolved picture of mixture toxicity and the accuracy of predictability with the CA and IA model in a complex organism, the embryos of the zebrafish, (2) determine whether mixture toxicity appearance and predictability follow a systematic pattern, and (3) give advice which of the above mentioned factors need to be considered as potential bias for mixture toxicity assessment in laboratory studies or in water quality regulation.

\section{Methods}

\section{Chemicals and mixtures}

The chemicals used for single substance and mixture toxicity studies were previously monitored and detected in several European surface waters. Busch et al. prioritized the identified chemicals with respect to their frequency of occurrence, hazard-quotients (HQ), and mode of action (MoA) [3]. Chemicals for this study were selected from a resulting top-30-compound-list. In total, 15 chemicals were selected and single substance toxicity determined. Table 1 shows the applied chemicals, their chemical structure, CAS registry number (CAS RN), usage or origin, their suspected $\mathrm{MoA}$, and calculated toxic ratio at $\mathrm{pH}=7.4$. Additional chemical information, such as $\log \mathrm{D}$ and solubility values, are listed in Additional file 1: Table S12.

To select the appropriate model for mixture toxicity prediction, it is believed important to know whether mixture constituents act similarly or independently. For instance, chemicals are considered to act similarly if they either exert unspecific toxicity or interfere with the same molecular target site, hence via the same MoA. For example, naproxen and diclofenac, two non-steroidal anti-inflammatory drugs (NSAIDs) exert their toxicity by inhibiting cyclooxygenase I and II (COX I and II) hence via the same MoA. However, chemicals that do not interact with specific targets, so called baseline toxicants, may also act similarly by inducing narcosis. This non-specific MoA is simply triggered by the intercalation of a chemical with the membrane, thus resulting in narcosis of the organism [28]. The level of accumulation and effect is directly dependent on the chemical's hydrophobicity. The calculation of the baseline toxicity of a chemical in zebrafish embryos (ZFE) was estimated by applying Eq. (1) according to [29]

$$
\log \left(\frac{1}{\mathrm{EC}_{50}[\mathrm{mM}]}\right)=\left(0.99 * \log K_{\mathrm{ow}}\right)-2.02 .
$$

The $\mathrm{EC}_{50}$ is defined by the concentration that causes an adverse effect for $50 \%$ of evaluated ZFE. The $\mathrm{EC}_{50}$ is dependent on the distribution coefficient between water and $\mathrm{n}$-octanol, the $\log K_{\mathrm{OW}}$. The $\log K_{\mathrm{OW}}$ values were taken from CHEMSPIDER and were derived from the prediction of $\mathrm{ACD} / \mathrm{labs}$ at a $\mathrm{pH}=7.4$ (see Additional file 1: Table S12).

Whether a chemical acts as a baseline toxicant or exerts a specific toxic effect can be estimated with the toxic ratio (TR, see Eq. 2) [30]. The TR is the quotient of the predicted to actually measured toxicity of a chemical:

$$
\mathrm{TR}=\frac{\mathrm{EC}_{50 \_ \text {pred }}}{\mathrm{EC}_{50 \_ \text {obs }}}
$$

where $\mathrm{EC}_{50 \_ \text {obs }}$ is the measured concentration of a chemical that exerts an adverse effect for $50 \%$ of the examined test organisms, whereas the $\mathrm{EC}_{50}$ pred is defined by its predicted counterpart, hence the $\mathrm{EC}_{50}$ value of a chemical calculated by QSAR models based on its $\log K_{\text {OW }}$ $(\mathrm{pH}=7.4)$ (see Eq. 1). According to Verhaar et al. [31] a $\mathrm{TR} \leq 10$ classifies a chemical to act as a baseline toxicant (inert or less inert chemicals), whereas chemicals with a TR $>10$ are assumed to have a specific mode of toxic action (reactive and specifically acting chemicals). Here, we also applied a $\mathrm{TR}=10$ as the threshold to separate non-specifically from specifically acting chemicals.

Based on specific research questions and underlying MoA of selected test chemicals, nine different mixtures were designed. Table 2 gives an overview of the analyzed mixtures (mixA-F), the number of compounds in the specific mixture, the examined exposure duration, the highest test concentration, and the selected dilution factor (the dilution factor was kept constant, consequently the mixture ratio (MR) also remained constant throughout one experiment).

MixB and mixC were designed to investigate whether the toxicity of a mixture is reliably predictable when consisting of only similarly (mixB) or dissimilarly acting components (mixC) in ZFE using the appropriate model. MixB consisted of five similarly acting components, namely bisphenol A, cyprodinil, diazinon, naphthalene, and triclosan. Next to known molecular interactions of these chemicals with individual biomolecules, the chemicals were expected to exert their toxicity via narcosis in the ZFE as the calculated TR was below ten. In contrast, the TR of chemicals applied in mixC exceeded the threshold value of ten and were thereof suspected to exert their toxicity via a specific MoA. To investigate the predictive power of the IA model, we selected five presumed specifically and independently acting substances, namely carbendazim, cyprodinil, diuron, diclofenac sodium salt, and genistein. Cyprodinil, however, represents an exception as its TR equals 9.34 and does, therefore, not imply a clear specific action according to our criterion. However, as cyprodinil is the only chemical with a $\mathrm{TR}<10$ in that mixture, even a narcotic MoA would be considered independent from the others. The 
Table 1 List of chemicals used in this study

\begin{tabular}{|c|c|c|c|c|c|}
\hline Substance & Structure & CAS RN & Usage/origin & Mode of action & Toxic ratio \\
\hline Benzo[a]pyrene & & $50-32-8$ & PAH, incomplete burning & $\begin{array}{l}\text { Epoxide reacts with DNA and produces carcino- } \\
\text { genic adduct }\end{array}$ & * \\
\hline Benzo[b]fluoranthene & & $205-99-2$ & PAH, incomplete burning & Mutagenic & * \\
\hline Bisphenol A & & 80-05-7 & Polymer synthesis & Binding to estrogen-related receptors & $5.18 \mathrm{E}-01$ \\
\hline Carbendazim & & $10605-21-7$ & Fungicide & Inhibitor of mitosis and cell division & $1.51 \mathrm{E}+03$ \\
\hline Chlorophene & & $120-32-1$ & Antiseptic drug, disinfectant & Unknown & $1.94 \mathrm{E}+00$ \\
\hline Cyprodinil & & $121552-61-2$ & Fungicide & Inhibition of methionine biosynthesis & $9.34 \mathrm{E}+00$ \\
\hline Diazinon & & $333-41-5$ & Insecticide & Inhibition of acetylcholinesterase & 8.17E-01 \\
\hline Diclofenac sodium salt & & $15307-79-6$ & Pharmaceutical & $\begin{array}{l}\text { Inhibition of cyclooxygenase I and II, non-steroi- } \\
\text { dal anti-inflammatory drug (NSAID) }\end{array}$ & $6.54 \mathrm{E}+02$ \\
\hline Diuron & & $330-54-1$ & Herbicide & $\begin{array}{l}\text { Inhibition of photosynthesis: blocking of plasto- } \\
\text { quinone binding site of PSII }\end{array}$ & $5.40 \mathrm{E}+03$ \\
\hline Genistein & & $446-72-0$ & Antineoplastic agent & $\begin{array}{l}\text { Inhibition of protein-tyrosine kinase and topoi- } \\
\text { somerase II: induction of G2-phase arrest in } \\
\text { human and mice }\end{array}$ & $2.21 E+02$ \\
\hline Naphthalene & & $91-20-3$ & Industry, insecticide & Epoxide induces hemolysis, induces narcosis & $1.20 E+00$ \\
\hline Naproxen sodium salt & & $26159-34-2$ & Pharmaceutical & $\begin{array}{l}\text { Inhibition of cyclooxygenase I and II, non-steroi- } \\
\text { dal anti-inflammatory drug (NSAID) }\end{array}$ & $1.09 \mathrm{E}+02$ \\
\hline Propiconazole & & 60207-90-1 & Fungicide & Inhibition of ergosterol biosynthesis & $5.34 \mathrm{E}-01$ \\
\hline Triclosan & & $3380-34-5$ & $\begin{array}{l}\text { Anti-infective agent, anti- } \\
\text { septic drug, disinfectant }\end{array}$ & $\begin{array}{l}\text { Binding of bacterial enoyl-acyl carrier protein } \\
\text { reductase enzyme }\end{array}$ & $8.85 E-01$ \\
\hline Triphenyl phosphate & & $115-86-6$ & Flame retardant, plasticizer & $\begin{array}{l}\text { Neurotoxicity, developmental toxicity, meta- } \\
\text { bolic disruption, endocrine activity, genotox- } \\
\text { icity and estrogenic activity }\end{array}$ & $1.56 \mathrm{E}+00$ \\
\hline
\end{tabular}

*No toxicity observed at measured concentrations

substances for mixB and mixC were further selected with respect to sufficiently distinguish the CA and IA prediction. Next, we were interested whether the developmental stage has impact on the accuracy of the CA and IA model. Therefore, we exposed the ZFE not only at the age of $0 \mathrm{hpf}$ (mixB.1 and mixC.1) but also at the age of $24 \mathrm{hpf}$ (mixB.2 and mixC.2). The comparability of these mixtures was achieved through an equitoxic mixture 


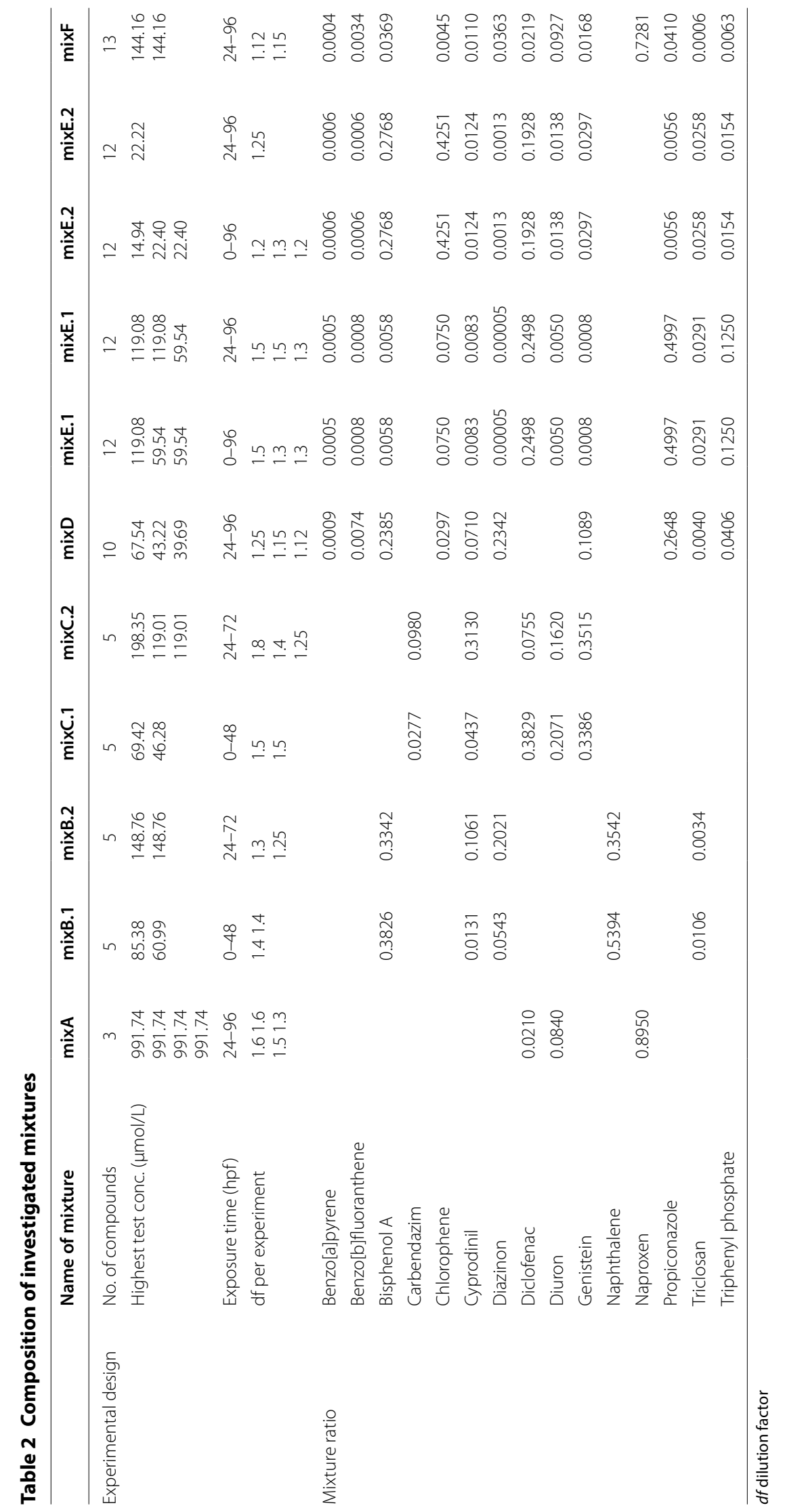


design and identical exposure durations. The anchoring point of the mixture design was based on the individual compounds $\mathrm{LC}_{10}$ values obtained from single substance toxicity testing for an exposure duration of $0-48 \mathrm{hpf}$ for mixB.1 and mixC.1 and 24-72 hpf for mixB.2 and mixC.2, respectively. This means that the mixture contained equally effective fractions of individual mixture components when exposed for either $0-48 \mathrm{hpf}$ (mixB.1 and mixC.1) or 24-72 hpf (mixB.2 and mixC.2) at a certain mixture concentration. As the toxicity obtained from single substance toxicity testing varied when the ZFE were exposed directly after hatching $(0 \mathrm{hpf})$ or in a later developmental stage (24 hpf), the MR of mixture components was adapted for each mixture.

MixA, mixD, and mixF were designed to specifically investigate the impact of (1) a rather high concentrated, simple mixture with clear expectations regarding mixture toxicity based on a previous single compound study [32] (mixA), (2) a mixture only containing chemicals below their individual effect threshold (mixD), and (3) a combination of mixA and mixD to study the combined effect of a mixture causing a specific effect behind a background of chemicals at low concentrations.

MixE was part of the inter-laboratory study published by Altenburger and colleagues [14]. In this study, mixE.1 was designed to have at least two bioactive compounds in each of the applied bioassays to guarantee detectability of mixture toxicity. MixE.2 contained the same components but at environmentally relevant concentrations. In that study, both mixtures were investigated for an exposure duration of 0-48 hpf and only mortality was determined with the ZFET (zebrafish embryo acute toxicity assay). In the current study, we expanded the dataset by elucidating the same mixtures for extended exposure durations of 0-96 hpf and 24-96 hpf and recorded not only lethal but also sublethal and teratogenic effects.

For all mixtures, the toxicity, hence lethal, sublethal, and teratogenic effects, were observed every $24 \mathrm{~h}$ within exposure period.

\section{Zebrafish maintenance and embryo collection}

Zebrafish maintenance was performed according to OECD 236 [33]. Here, a breeding stock of unexposed zebrafish (previously obtained from a local OBI hardware store) was used for egg production and the fish were maintained at a continuous photoperiod of 14:10 h light:dark. The feeding was conducted twice a day either with commercial dry food flakes or Artemia sp. and spawning initiated by a combination of light stimulus and a spawning mockup equipped with artificial plants. Eggs were collected $1 \mathrm{~h}$ after spawning and fertilized and non-fertilized eggs were immediately differentiated using a visualizing microscope (Olympus IX70).
Only fertilized, symmetrical dividing, and undamaged eggs within a cell stage of 4-126 cells were selected and stored into a petri-dish filled with oxygenated ISO standard dilution water (ISO water: ISO 7346-3; $79.99 \mathrm{mM}$ $\mathrm{CaCl} 2 \cdot 2 \mathrm{H} 2 \mathrm{O}, 20.00 \mathrm{mM} \mathrm{MgSO} 4 \cdot 7 \mathrm{H} 2 \mathrm{O}, 30.83 \mathrm{mM}$ $\mathrm{NaHCO}, 3.09 \mathrm{mM} \mathrm{KCl}, \mathrm{pH}=7.4 \pm 0.1)$. Subsequently, eggs were either exposed to chemicals straight after embryo selection (approximately within $2 \mathrm{~h}$ after fertilization, further referred as $0 \mathrm{hpf}$ ) or stored in $2 \mathrm{~mL}$ ISOwater per embryo at $28{ }^{\circ} \mathrm{C}$ until exposure start, i.e., 50 embryos were stored in $100 \mathrm{~mL}$ of ISO-water for $24 \mathrm{~h}$. In this study, embryos were exposed at two developmental stages, thus either at the age of $0 \mathrm{hpf}$ or $24 \mathrm{hpf}$.

\section{Test conditions, exposure solutions and quality control}

ISO water was aerated with oxygen 1 day prior to the experiment, a minimum oxygen saturation of $80 \%$ was verified using WTW Oxi 340 Oximeter and a $\mathrm{pH}$ of $7.4 \pm 0.1$ adjusted with $\mathrm{HCl} / \mathrm{NOH}$ and WTW SenTix Mic. For single substance toxicity assessment, a stock solution of the desired chemical in ISO-water was prepared 1 day prior to the experiment and directly applied as highest test concentration. In case, chemicals were not easily soluble in ISO-water, a co-solvent was used. Therefore, a 1000 fold stock solution in either methanol or DMSO was prepared, stored at $4{ }^{\circ} \mathrm{C}$ or room temperature until usage and diluted on the day of experiment to the desired test concentration. At this, the solvent content did not exceed a maximum content of $0.1 \%$ solvent/ ISO-water $(\mathrm{V}: \mathrm{V})$ in all tested concentrations. For mixture toxicity testing, a 1000-fold concentrated mixture stock solution was prepared in methanol by mixing high concentrated single substance stock solutions in specific mixture ratios (MRs). The MR describes the proportion of one component that is present in a mixture relative to the total molarity of this mixture. The total molarity is calculated from the entirety of all single components present in their specific concentrations. At the day of experiment, the highest concentration of either single substance or mixture was diluted to 10-13 serial dilutions (constant dilution factor) by adding ISO-water containing the same solvent fraction as the highest concentration. The control solution consisted of ISO-water containing the same amount of solubilizing agent as the treatment solutions $(0.1 \% \mathrm{~V}: \mathrm{V})$. For detection of effects induced by controls, six technical replicates were used, whereas three technical replicates were conducted to analyze the effects induced by treatment solutions. For each replicate, three embryos were exposed to $6 \mathrm{~mL}$ of either treatment or control solution and incubated for desired exposure time in $7.5 \mathrm{~mL} \mathrm{GC}$ vials (VWR International) closed with aluminium lid and aluminium coated septum (Supelco Analytical). Incubation was conducted 
at $26{ }^{\circ} \mathrm{C}$ with a $12: 12 \mathrm{~h}$ light:dark photoperiod and vials shaken at $75 \mathrm{rpm}$ using a horizontal agitator (Edmund Bühler GmbH, SM-30 control). The toxicity tests of individual chemicals and mixtures were repeated at different days and concentrations adapted to obtain sufficiently resolved concentration-response curves (CRC), respectively. A test was classified valid if less than $20 \%$ of control embryos appeared with apical effects. Additionally, exposure solutions of the negative controls as well as those with the highest test concentration were examined for $\mathrm{pH}$ level and oxygen content at the first and last day of exposure. For complete validation of the test, a pH level between 6 and 8 and a minimum oxygen level of $60 \%$ was required at examined time points.

\section{Toxicity measurement of individual chemicals and mixtures}

Single substance and mixture toxicity were analyzed in a similar and comparable way. The toxicity of single substances was determined after exposing ZFE for 0-96 hpf and 24-96 hpf to all selected chemicals, respectively (15 in total, Table 1). Effects were recorded every $24 \mathrm{~h}$ within the exposure duration. Specific exposure durations and starting points ( $0 \mathrm{hpf}$ or $24 \mathrm{hpf})$ were selected to determine the toxicity of the mixtures. For both, single substance and mixture toxicity determination, 23 integral effects, classified in lethal, sublethal, and teratogenic (see Additional file 2: Table S1), were recorded using a dissecting microscope $(5 \times$, Olympus IX70). In this study, lethal describes all effects that imply the death of an organism (e.g., coagulation, missing heartbeat [33]), whereas sublethal and teratogenic effects are defined by effects which lead to a change in development and fitness of the organism or to malformations of the ZFE (e.g., change in blood circulation or frequency of heartbeat, formation of edema, or malformation of head, yolk, or tail, etc.), respectively. Additionally, abnormal hatching behavior was detected by determining hatching rates. Normally developed zebrafish larvae would hatch at 72 hpf. The raw data of lethal and total effect determination as well as hatching rates are listed in Additional file 3: Table S13. CRC were calculated separately for lethal and total (lethal + sublethal + teratogenic) effects by applying a Hill four-parameter model (Eq. 3) using the drc package, $\mathrm{R}$ (version 3.4.4) [34]:

$$
y=y_{0}+\frac{\left(a * x^{\mathrm{b}}\right)}{\left(c^{\mathrm{b}}+x^{\mathrm{b}}\right)},
$$

where $y$ refers to the level of lethal or total effects, $a$ to the minimum effect (0) and $y_{0}$ to the maximum effect (1). $c$ further refers to the $\mathrm{LC}_{50}$ or $\mathrm{EC}_{50}$ value, $b$ to the slope of the tangent at the inflection point and $x$ to the related concentration. For mixture toxicity analysis, we further derived three effect levels $\left(\mathrm{LC} / \mathrm{EC}_{10,50,90}\right)$ from mixture CRCs. This was done to address the potency of a mixture and the potential impact of mixture potency to model accuracy. For example, a mixture that induces an effect in $10 \%$ of examined organisms is less potent than a mixture that induces effects in $90 \%$ of the organisms. The potency of a mixture is linked to the composition of a mixture, hence the amount, concentration, and toxic potential of present components and their ability to induce a combined effect.

In conclusion, single substance and mixture toxicity was assessed in a concentration (10-13 dilutions of exposure solution), time (every $24 \mathrm{~h}$ within two exposure periods (0-96 hpf and 24-96 hpf)), effect type (23 quantal effects categorized in lethal and sublethal or teratogenic), and mixture potency resolved manner $\left(\mathrm{LC} / \mathrm{EC}_{10,50,90}\right)$. This approach allowed us to investigate the impact of mixture potency, exposure duration, and phenotype to mixture toxicity prediction quality of the models.

\section{Data analysis and regression models}

For mixture toxicity prediction, the CRC of single substances (ss_CRC) were additionally modeled using a "best-fit" approach. For that purpose, two non-linear models, the Logit (Table 3, Eq. 4) and Weibull model (Table 3, Eq. 5), were fitted to the experimental data using a maximum-likelihood approach and the best fitting model selected on the Aikaike Information Criterion (AIC). A smaller AIC indicates a better fit.

Where $\theta_{\min }$ is the minimum effect of 0 and $\theta_{\max }$ the maximum effect of 1 . Furthermore, $\theta_{1}$ describes the location and $\theta_{2}$ the steepness of the curve. The different regression models were fitted using the software $\mathrm{R}$ (version 3.4.4) and the package bbmle (Ben Bolker and R, Development Core, Team (2017). bbmle: Tools

Table 3 Regression models used for calculating the concentration-response relationships

\begin{tabular}{ll}
\hline Regression model & Formula \\
\hline Logit & $E=\theta_{\min }+\frac{\theta_{\max }-\theta_{\min }}{1+\exp \left(-\theta_{1}-\theta_{2} \log _{10}(\operatorname{conc} .)\right)}$ \\
Weibull & $E=\theta_{\min }+\left(\theta_{\min }-\theta_{\max }\right) *\left(1-\exp \left(-\exp \left(\theta_{1}+\theta_{2} * \log _{10}(\right.\right.\right.$ conc.) $)$ \\
\hline
\end{tabular}


for General Maximum-Likelihood Estimation. R package version 1.0.20., https://CRAN.R-project.org/packa $\mathrm{ge}=\mathrm{bbmle})$. The parameter of all concentration-response curves are summarized in Additional file 2: Tables S2-5.

\section{Mixture toxicity prediction}

We estimated the toxicity of all mixtures with two models, the concept of Concentration Addition (CA) and Independent Action (IA). By the definition of the concepts, CA predicts the toxicity of mixture containing similarly acting substances, whereas the IA model predicts the toxicity of mixtures that are composed of dissimilarly acting components.

The CA concept is formalized in Eq. (6):

$$
\mathrm{EC}_{\mathrm{x}, \text { mix }}=\left(\sum_{i=1}^{n} \frac{p_{i}}{\mathrm{EC}_{\mathrm{xi}}}\right)^{-1},
$$

where $\mathrm{EC}_{\mathrm{x} \text {,mix }}$ is the total concentration of the mixture provoking $x \%$ effect; $\mathrm{EC}_{\mathrm{xi}}$ is the concentration of $i$ th component provoking the $\mathrm{x} \%$ effect, when applied individually, and $p_{i}$ denotes the fraction of component $\mathrm{i}$ in the mixture. The calculation of total mixture concentrations for various effect levels lead to a complete iteration of an expected CRC $[9,26]$.

The IA concept is formalized in Eq.(7):

$$
\mathrm{E}\left(\mathrm{C}_{\mathrm{mix}}\right)=1-\prod_{i=1}^{n}\left[1-\mathrm{E}\left(\mathrm{C}_{\mathrm{Si}}\right)\right],
$$

where $\mathrm{E}\left(\mathrm{C}_{\mathrm{Si}}\right)$ are the effects of the single substances, $n$ the number of individual components, and $\mathrm{E}\left(\mathrm{C}_{\text {mix }}\right)$ is the total effect of the joint mixture $[11,35]$.

According to the mathematical formalizations of these two concepts, the CA concept implies that every toxicant in any concentration contributes, in proportion to its toxic unit, to the overall combined effect of a mixture $[9,10]$. In contrast, the IA model implies that only mixture constituents that are present in concentrations higher than their individual effect threshold (i.e., lowest observed effect concentrations) are able to contribute to the combined effect of a mixture $[10,11]$.

\section{Mixture toxicity analysis and comparison of measured with predicted toxicity}

To determine the prediction accuracy, we compared the observed toxicity to the prediction by calculating the prediction deviation ratio (PDR, Eq. 8). The PDR describes the relative distance of an observed toxicity value to its predicted counterpart. For example, a correct estimation of mixture effect is indicated by a PDR of 1 , whereas an underestimation of effect would be indicated by a $\mathrm{PDR}<1$. Consequently, a PDR $>1$ describes an overestimation of effect:

$$
\mathrm{PDR}=\frac{y_{i}}{x_{i}}=\frac{\mathrm{EC}_{\mathrm{pred}}}{\mathrm{EC}_{\mathrm{obs}}} .
$$

PDR represents the distance of a certain measured effect value $x_{i}\left(\mathrm{EC}_{\mathrm{obs}}\right)$ in comparison to its predicted counterpart $y_{i}\left(\mathrm{EC}_{\mathrm{pred}}\right)$. To facilitate data interpretation, the $\log _{2}$ values of PDR are applied for illustration.

\section{Results}

In this study, we investigated the accuracy of two mixture toxicity prediction models, the model of Concentration Addition (CA) and Independent Action (IA). We analyzed whether certain experimental and analysis parameters, such as the mixture design, exposure duration, phenotype, and mixture potency influence the predictability of mixture toxicity. The approach and the analysis strategy are summarized in Fig. 1. Firstly, we compared the model accuracy of the CA and IA model in general. Therefore, we investigated whether the IA model reliably predicts the toxicity induced by a mixture containing only dissimilarly acting components in a complex organism, the embryos of zebrafish (ZFE). The same was done for the CA model with a mixture that only contained suspected similarly acting components (Fig. 1a). We also investigated whether the developmental stage ( 0 or $24 \mathrm{~h}$ post fertilization (hpf)) at which the ZFE were exposed to the mixtures influenced the predictability of mixture toxicity. Secondly, we extended mixture toxicity analysis and examined the potential impact of exposure duration, phenotype, and mixture potency on mixture toxicity predictability (Fig. 1b). Therefore, we observed lethal, sublethal, and teratogenic effects induced in ZFE after exposure to nine different mixtures for varying exposure durations (24, 48, 72, $96 \mathrm{~h}$ post exposure (hpe)). Overall, we analyzed 31 different exposure scenarios from which we obtained 28 concentration-response curves (CRCs) for lethal and 31 CRCs for total (lethal + sublethal + teratogenic) effects. We further calculated $\mathrm{LC} / \mathrm{EC}_{\mathrm{x}}(x=10$, $50,90)$ values from each mixture CRC. Consequently, we derived 177 toxicity values from effect observations. These toxicity values were further compared to their counterparts predicted with CA and IA by calculating the prediction deviation ratio (PDR). Thirdly, we analyzed the general appearance and time dependence of mixture toxicity concentration-response curves (mix CRCs) (Fig. 1c). Finally, we studied the induction of combined toxic effects in ZFE after exposure to mixtures in which the components were present in concentrations that failed to evoke individual toxicity. This phenomenon 


\section{Analysis strategy}

a Model comparison - mixtures containing dissimilarly or similarly acting components

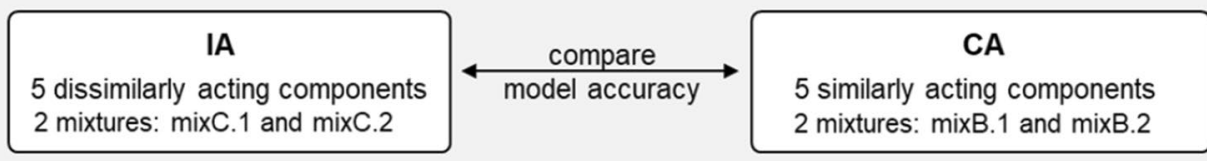

b Model performance - influence of experimental parameters on accuarcy of CA and IA model

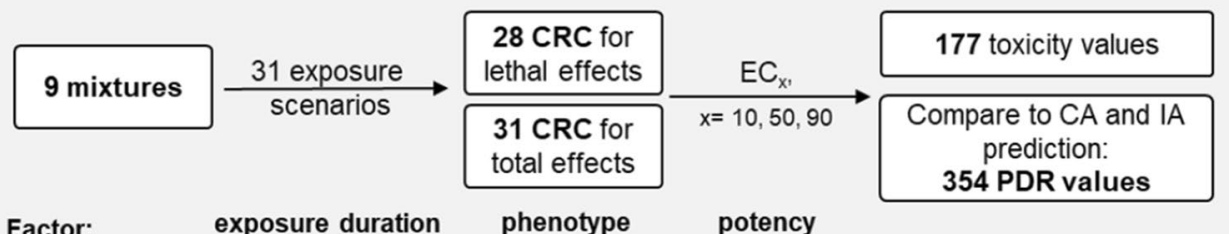

Factor: exposure duration phenotype potency

C Analysis of mixture CRC slopes and time dependence

d Principle 'something from "nothing"'

Fig. 1 Workflow and outline of the mixture toxicity analysis strategy

is considered as the principle 'something from "nothing"' (Fig. 1d).

\section{Example mixture composed of dissimilar acting components}

The workflow of mixture toxicity prediction and observation was similar for all investigated mixtures. This workflow is shown on the example of mixC.1 and selected results for mixC. 1 are depicted in Fig. 2.

MixC.1 was composed of five suspected independently acting substances (see Table 2), namely carbendazim (triangle), cyprodinil (circle), diclofenac (diamond), diuron (star), and genistein (square). First, the toxicity of single components was determined by exposing ZFE at a certain age (here: $0 \mathrm{hpf}$ ) to the respective chemical (Fig. 2a). After a defined exposure period (here: $24 \mathrm{~h}$ and $48 \mathrm{~h}$ ), specific endpoints (here: lethal effects) were observed and respective CRCs modeled. Figure $2 \mathrm{~b}$ shows the CRC for single substances (ss_CRC) obtained after $24 \mathrm{~h}$ of exposure (hpe; solid, black line and pink symbols) and 48 hpe (dashed, grey line and purple symbols). Carbendazim, cyprodinil, and genistein show no time dependent toxicity, whereas lethal concentrations of diclofenac and diuron are decreasing over time $\left(\mathrm{LC}_{50 \_ \text {diclofenac_oto24hpf }}=133.15 \mu \mathrm{M}, \mathrm{LC}_{50 \_d i c l o f e n a c \_0 t o 48 \mathrm{hpf}}=\right.$ $15.84 \mu \mathrm{M})$. In this case, the ss_CRC obtained after a certain exposure period (here: $48 \mathrm{hpe}$ ) were further applied to design a mixture in which all components were present in equally effective fractions (here: individual $\mathrm{LC}_{10}$ ) when exposed to the highest mixture concentration and respective exposure period. The mixture ratios (MR) and ss_CRCs were used to predict mix_CRCs with the CA (light blue) and IA model (yellow) (Fig. 2c: $24 \mathrm{~h}$ (pink), Fig. $2 \mathrm{~d}: 48 \mathrm{~h}$ (purple)). Due to time dependence of single substance toxicity, the impact of a certain chemical to a combined effect may vary over time. For example, diclofenac (diamond) showed the least toxic influence regarding the short exposure period and the most toxic influence regarding the longer exposure period. Subsequently, ZFE were exposed to the designed mixture, lethal effects recorded, and mixture toxicity observed (Fig. 2e). The mixture toxicity observation was performed analogously to the single substance toxicity tests, hence the same exposure periods and endpoints were considered. Figure 2f shows the mix_CRCs obtained after 24 hpe (pink) and 48 hpe (purple). Here, the mixture toxicity

\footnotetext{
(See figure on next page.)

Fig. 2 Experimental workflow of mixture analysis, exemplarily shown for mixC.1. Single substance toxicity observation. a Exposure of ZFE to different concentrations of single substances and effect determination at different time points. b Single substance concentration-response curve (ss_CRC) for all mixture components (triangle: carbendazim, circle: cyprodinil, diamond: diclofenac, star: diuron, square: genistein) obtained after an exposure duration of 24 hpe (pink) and 48 hpe (purple). Mixture toxicity prediction based on ss_CRCs (black) with the CA (light blue) and the IA (yellow) model, respectively, for the exposure durations of 24 hpe (c, pink), and 48 hpe (d, purple). e Exposure of ZFE to different concentrations of the mixture (fixed MR) and effect determination at different time points. f Observed mixture toxicity (mix_CRC) at two time points. Comparison of observed (black) and predicted mixture toxicity (light blue: CA, yellow: IA) for the 24 hpe (g, pink) and 48 hpe (h, purple) exposure duration
} 

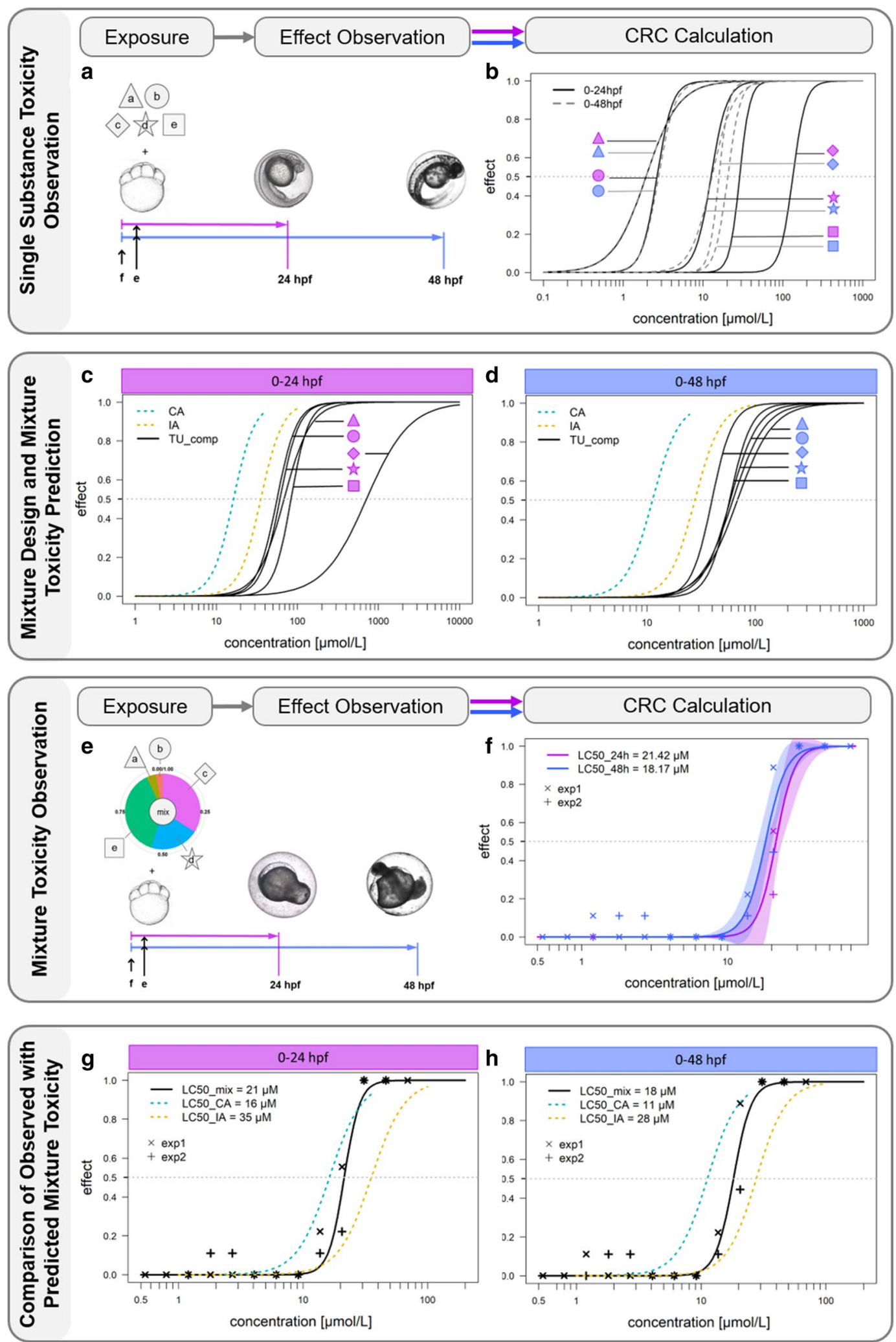
showed no significant variation upon different exposure durations $\left(\mathrm{LC}_{50 \_24 \mathrm{hpe}}=21 \mu \mathrm{M}, \mathrm{LC}_{50 \_48 \mathrm{hpe}}=18 \mu \mathrm{M}\right.$, Additional file 2: Table S8). Finally, we compared the observed with the predicted mixture toxicity. Figure $2 \mathrm{~g}$ and $\mathrm{h}$ show the observed mix_CRCs (solid, black line) vs. the predicted CRCs calculated with the CA (dashed, yellow line) and IA model (dashed, light blue line), respectively, for the two different exposure durations (Fig. 2g: 24 hpe (pink), $2 \mathrm{H}$ : 48 hpe (purple)). We found that the experimentally determined mixture toxicity was not fully reflected by the IA prediction but fell in the concentration-effect space that is spanned between both models. This concentration-effect space is further termed as prediction window. However, the observed mix_CRC approximates towards the IA curve at low effect concentrations, whereas the CA model results to better reflect the mix_CRC at higher effect concentrations. Both mix_CRCs are steep and quotients of $\mathrm{LC}_{75}$ to $\mathrm{LC}_{50}$ obtained after 24 and $48 \mathrm{~h}$ of exposure are relatively small $\left(\mathrm{LC}_{75} / \mathrm{LC}_{50}(24 \mathrm{hpe})=1.17, \mathrm{LC}_{75} / \mathrm{LC}_{50}(48 \mathrm{hpe})=1.18\right.$, Additional file 2: Table S8). Hence a concentration increase of less than $20 \%$ results in double the effect.

A second mixture with the same components but different MRs (mixC.2) was investigated at a later developmental stage of the ZFE and exposure was started at 24 hpf. Again, the observed mixture toxicity fell into the prediction window (Additional file 2: Figure S3). The mix_CRCs also occurred to be steep $\left(\mathrm{LC}_{75} / \mathrm{LC}_{50}\right.$ (24 hpe $)=1.10, \mathrm{LC}_{75} / \mathrm{LC}_{50}(48 \mathrm{hpe})=1.15$, Additional file 2 : Table S8). However, the time dependence of toxicity was stronger in ZFE exposed at a later developmental stage $\left(\mathrm{LC}_{50 \_24 \mathrm{hpe}}=83 \mu \mathrm{M}, \mathrm{LC}_{50 \_48 \mathrm{hpe}}=44 \mu \mathrm{M}\right.$, Additional file 2: Table S11).

\section{Example mixture of similarly acting components}

Next, we investigated a mixture consisting of suspected similarly acting components (mixB). Again, ZFE in two different developmental stages were exposed (from $0 \mathrm{hpf}$ : mixB.1 and 24 hpf: mixB.2, Table 2) to two similar mixtures with adopted MRs. The obtained mix_CRCs and their comparison to predictions are shown in Additional file 2: Figure S2. The mix_CRCs are again located within the prediction window but observations were much closer to the CA prediction. Regarding curve steepness and time dependence of toxicity, $\operatorname{mixB}$ showed a similar trend as previously described for mixC. No significant difference between the $24 \mathrm{~h}$ and a $48 \mathrm{~h}$ exposure duration was observed for the early treatment period (mixB.1, $\mathrm{LC}_{50 \_24 \mathrm{hpe}}=35 \mu \mathrm{M}, \mathrm{LC}_{50 \_48 \mathrm{hpe}}=34 \mu \mathrm{M}$ ), whereas the late exposure start resulted in an increase in toxicity over time $\left(\right.$ mixB.2, LC50 $\left.24 \mathrm{hpe}=108 \mu \mathrm{M}, \mathrm{LC}_{50 \_48 \mathrm{hpe}}=77 \mu \mathrm{M}\right)$. Again, steep mix_CRC were detected for all respective mixtures $\left(\operatorname{mixB} .1: \mathrm{LC}_{75} / \mathrm{LC}_{50}(24 \mathrm{hpe})=1.21, \mathrm{LC}_{75} / \mathrm{LC}_{50}\right.$
$(48$ hpe $)=1.15$, Additional file 2: Table $\mathrm{S} 8$ and mixB.2 $\mathrm{LC}_{75} / \mathrm{LC}_{50}(24 \mathrm{hpe})=1.04, \mathrm{LC}_{75} / \mathrm{LC}_{50}(48 \mathrm{hpe})=1.06$, Additional file 2: Table S11).

To investigate the generality of the obtained results, we extended the mixture toxicity analysis to results obtained after exposure of ZFE to nine different mixtures (Table 2). All integrated mixture experiments were analyzed by applying the previously described workflow.

\section{Mixture toxicity falls within the prediction window that is spanned by the CA and IA model}

We compared the experimentally determined mixture effect concentrations with their predicted counterparts for all tested mixtures. Figure 3 depicts the $\log _{2}$ of the prediction deviation ratio (PDR) of observed to predicted effect concentrations that induce $50 \%$ of an effect in ZFE. The figure shows the relative distance of measured mixture toxicity to the prediction that is based on the CA (lightblue) and IA (yellow) model for lethal (left, $\mathrm{LC}_{50}$ ) and total (lethal + sublethal + teratogenic) effects (right, $\mathrm{EC}_{50}$ ). For three exposure scenarios no lethal effects could be observed for the tested concentrations (0-24_mixE.2, 24-48_mixE.2, and 24-48 h_mixF). The observed mixture toxicity was located within the prediction deviation range between the CA and IA estimations in 51 out of 59 cases $(86 \%)$ or was even underestimated by both models $(8 / 59,14 \%)$. As only underestimation of mixture toxicity occured, a mixture toxicity value was determined as being located within the prediction window when the PDR calculated with the CA model (CA is predicts the highest toxicity values in all cases) didn't exceed 1.1 (PDR_CA $<1.1$, deviation of $10 \%$ allowed). Similar findings were obtained when comparing not only $\mathrm{EC}_{50}$ but also $\mathrm{EC}_{10}$ and $\mathrm{EC}_{90}$ values (Additional file 2: Figure S10). All 177 calculated PDRs are listed in Additional file 2: Table S6. In total, 149 out of 177 (84\%) inspected mixture effect concentration values were located within the prediction window, whereas in 28 out of 177 cases, mixture toxicity was underestimated with both models. We observed that lower effect concentrations seem to be better predictable with the IA concept, whereas the CA model seems to better estimate the toxicity of higher effect concentrations. Additionally, high effect values seem even more likely to be underestimated by the applied prediction models. Half of the cases at which the mixture was more potent than predicted derive from comparisons of high effect values $\left(\mathrm{EC}_{90}\right.$ or $\left.\mathrm{LC}_{90}\right)$ to their predicted counterparts $(14 / 28)$.

\section{Mixture toxicity prediction accuracy depends on mixture potency and exposure duration}

To analyze whether and to which extent the predictability of mixture effects in the ZFE is dependent on 


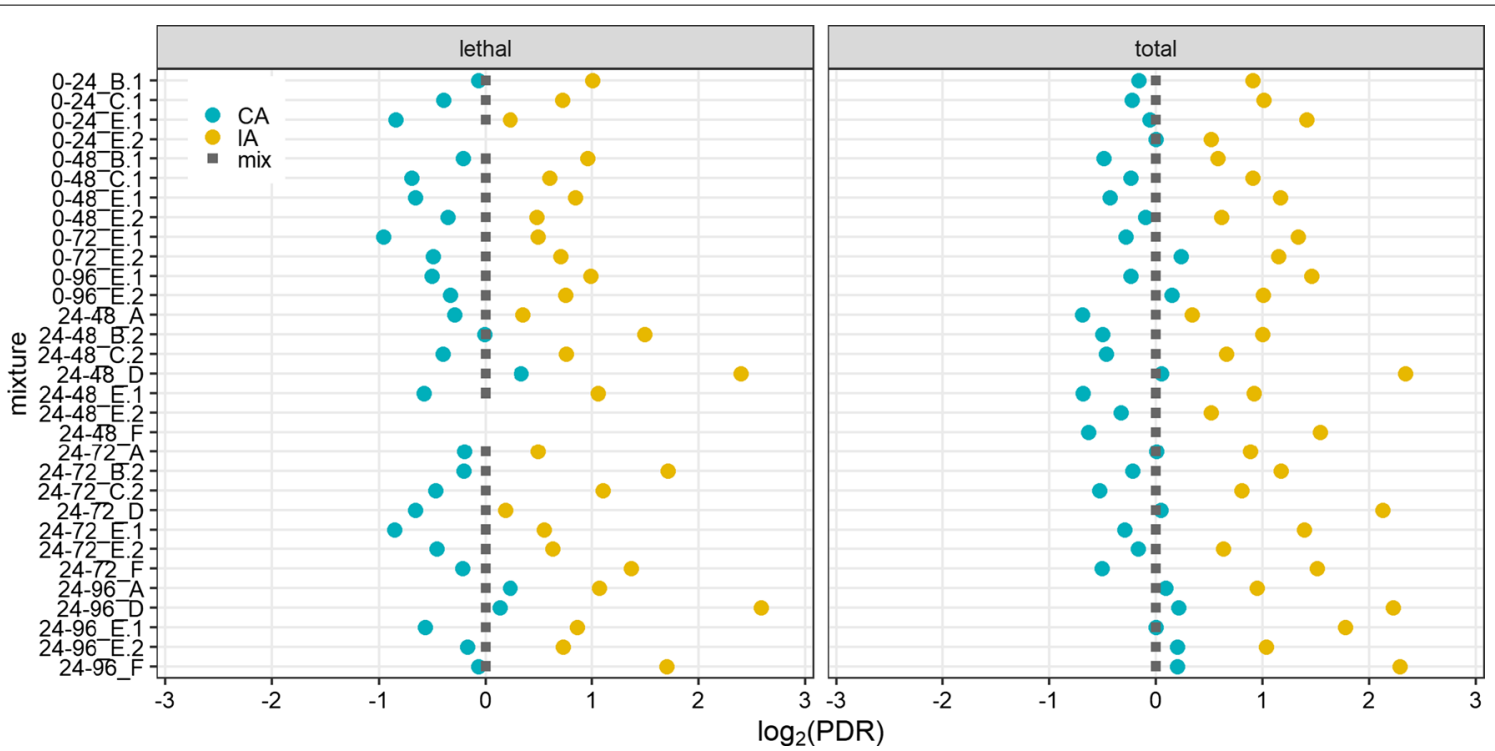

Fig. 3 Comparison of observed mixture toxicity to predicted mixture toxicity. Shown are the $\log _{2} P D R$ of $E C_{50}$ values that were predicted with the CA model (blue) and IA model (yellow) vs. respective experimentally determined $\mathrm{EC}_{50}$ values (grey). Left panel: lethal effects. Right panel: total (lethal + sublethal + teratogenic) effects

experimental and analysis variables, such as exposure duration, considered phenotype, and mixture potency, we compared PDRs for these measures. Therefore, we looked at the toxicity values obtained after four exposure durations (24, 48, 72, 96 h), two effect types (lethal and total) that represent different phenotypes and three effect levels $\left(\mathrm{EC}_{x}, x=10,50,90\right)$ that represent different mixture potencies.

Overall, we compared 177 toxicity values (28 lethal, 31 total, 3 mixture potencies) obtained from measured mixture toxicity testing to their counterparts that were predicted with the CA and IA model. This resulted in 354 PDR values that could be considered (177 for each model, Additional file 2: Table S6). Figure 4 illustrates the distributions of the $\log _{2}$ of these PDRs with regard to certain analysis parameters. Figure 4 a depicts the distribution of all 354 determined PDRs (grey bars), whereas the colored density plots show the distributions of PDRs for CA (light blue) and IA (yellow), respectively. A further detailed resolution is shown in Fig. 4b, where the PDR distributions obtained for lethal and total effects were plotted separately. Similar to the results shown in Fig. 3, we see that, on average, the measured mixture toxicity (black line) was again located within the prediction window spanned between the CA and IA estimations even when not only $\mathrm{EC}_{50}$ values but also $\mathrm{EC}_{10}$ and $\mathrm{EC}_{90}$ values were considered. Comparing the means of the $\log _{2} \mathrm{PDRs}$ for $\mathrm{CA}$ (dashed line, light blue, mean_log ${ }_{2} \mathrm{PDR}_{\mathrm{CA} \_ \text {lethal }}=-0.28$, Additional file 2: Table S7) and IA model (dashed line, yellow, mean_log ${ }_{2} \mathrm{PDR}_{\mathrm{IA} \_ \text {lethal }}=1.06$, Additional file 2: Table S7) reveals that experimentally determined mixture toxicity values were rather matching with the predicted toxicity values calculated by the CA model. This became even more apparent when total effect concentrations are considered (Fig. 4b, mean $\_\log _{2} \mathrm{PDR}_{\mathrm{CA} \_ \text {total }}=-0.20$, mean_log $\left.\log _{2} \mathrm{PDR}_{\mathrm{IA} \_ \text {total }}=1.17\right)$. The PDR distributions respecting the three effect levels $\left(\mathrm{EC}_{10}\right.$ : light blue, $\mathrm{EC}_{50}$ : yellow, $\mathrm{EC}_{90}$ : pink) are depicted in Fig. $4 \mathrm{c}$ (all data) and Fig. $4 \mathrm{~d}$ (differentiated with respect to the applied models (left: CA, right: IA)). Inspecting the predictability of different effect levels revealed that the highest model accuracy was obtained when high effect concentrations were predicted with the CA model. On average, here, the observation only deviated from the prediction by a factor of 1.3 (mean_log ${ }_{2} \mathrm{PDR}_{\mathrm{CA} \_\mathrm{EC} 90}=-0.146$ ). On a single model basis, the IA model achieved highest

(See figure on next page.)

Fig. 4 Distributions of prediction deviation ratios for mixture toxicity determined with the ZFE. Distributions are grouped by a prediction models, $\mathbf{b}$ prediction models and phenotype, $\mathbf{c}$ mixture potency, $\mathbf{d}$ mixture potencies and prediction models, $\mathbf{e} \mathrm{EC}_{50}$ and phenotype, $\mathbf{f}$ by $\mathrm{EC}_{50}$ and phenotype and prediction model, $\mathbf{g} \mathrm{EC}_{50}$ and exposure duration, and $\mathbf{h} \mathrm{EC}_{50}$ and exposure duration and prediction model. Colored, dashed lines: Mean $\log _{2}$ PDRs for respective groups 

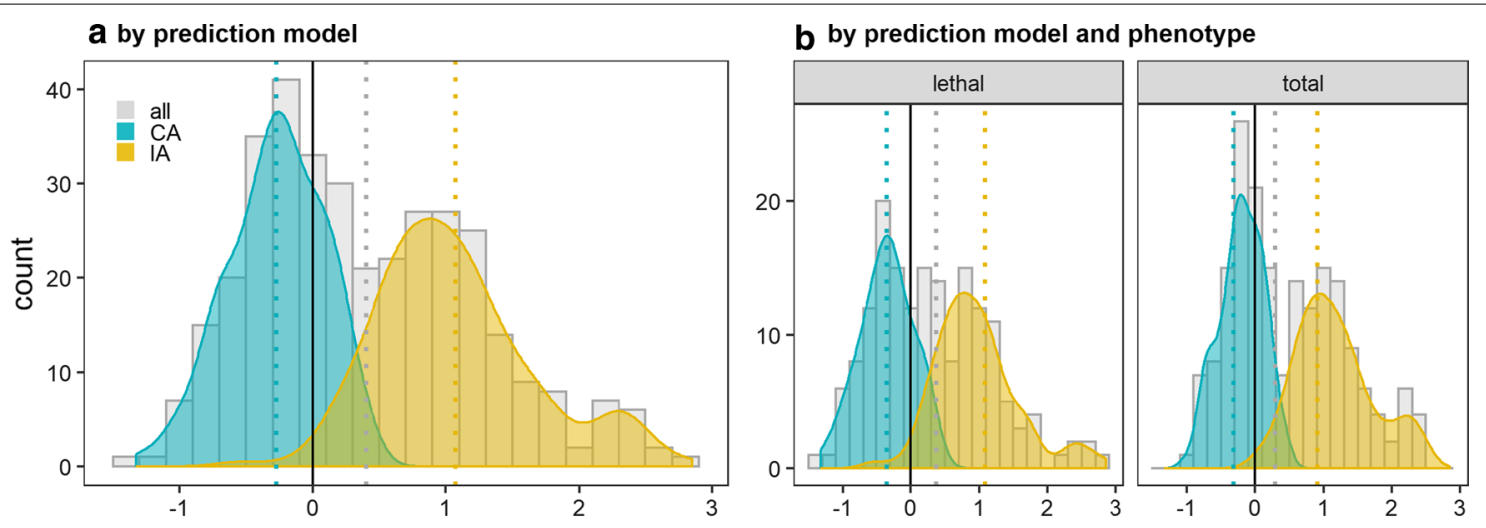

C by mixture potency

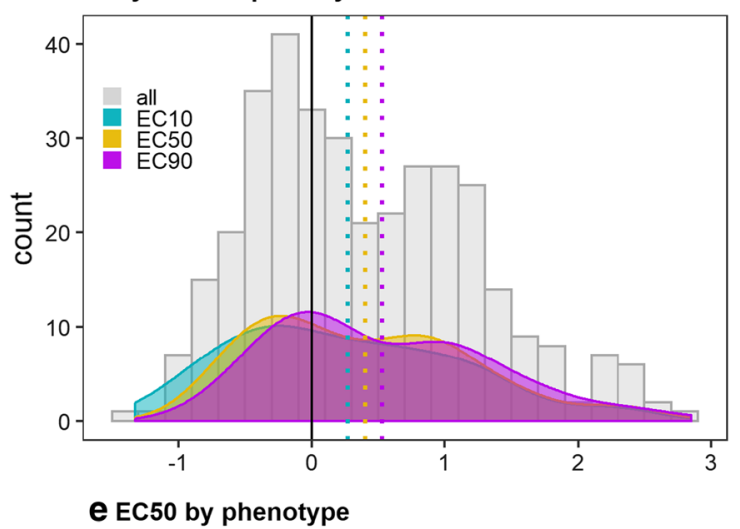

d by mixture potency and prediction model
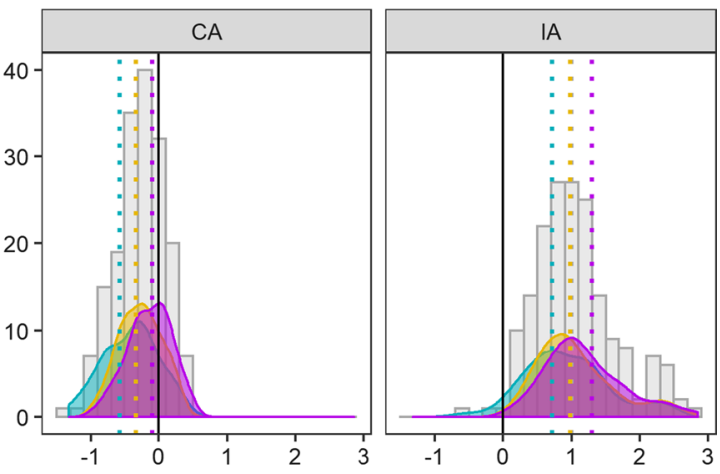

f EC50 by phenotype and prediction model
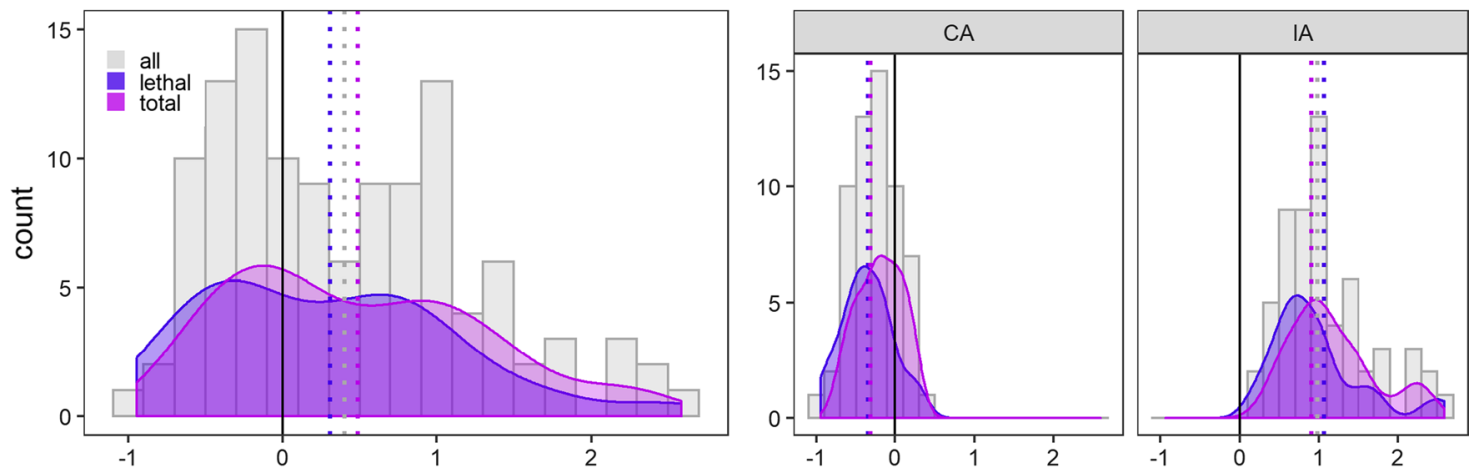

\section{g EC50 by exposure duration}

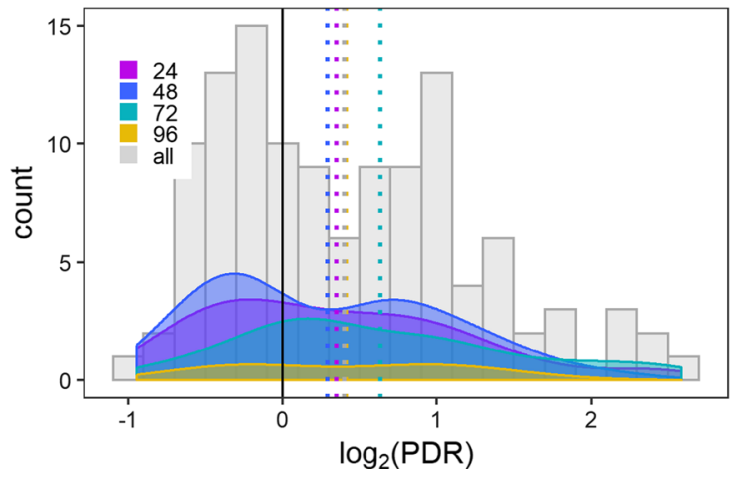

\section{h EC50 by exposure duration and prediction model}

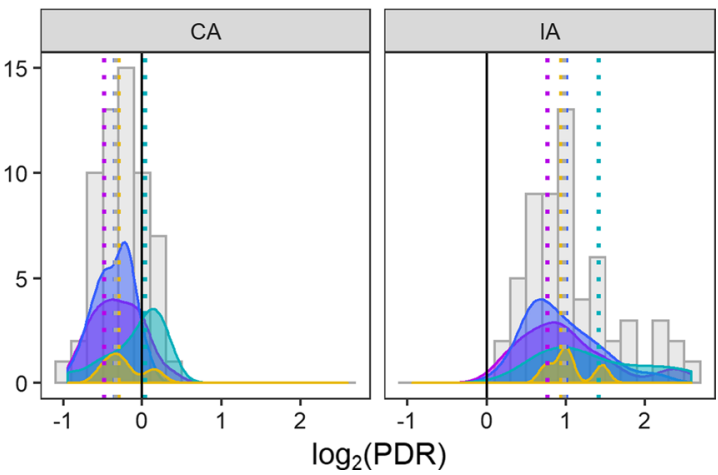


prediction accuracy when low mixture effects were estimated (mean_log ${ }_{2} \mathrm{PDR}_{\mathrm{IA} \_\mathrm{EC} 10}=0.941$, mean_log $2 \mathrm{PDR}_{\mathrm{IA}}$ $\mathrm{EC}_{0}=1.057$, mean_log $\left.{ }_{2} \mathrm{PDR}_{\mathrm{IA} E \mathrm{EC} 90}=1.178\right)$. However, comparing both models, the CA model was still closer to the observations than the IA predictions. This did not consolidate the trend described in Figs. $2 \mathrm{~g}$ and $\mathrm{h}$ and 3, where IA seemed to better predict low levels of mixture effects.

For this reason and because the $\mathrm{EC}_{50}$ values were the most robust ones for prediction, we specified further mixture analysis on the $\mathrm{EC}_{50}$ values for lethal and total effects. In a next step we compared the PDR distributions for lethal and total effects, as well as for different exposure durations based on $\mathrm{EC}_{50}$ values (Fig. $4 \mathrm{e}, \mathrm{f}, \mathrm{h}$ ). In Fig. $4 \mathrm{e}$ it is shown that the predictions were not significantly influenced by the respective considered effect type (lethal or total) as PDRs were similarly distributed for both effect types (purple: lethal, pink: total). Again, the PDRs for CA were smaller than for IA for both types of effect (Fig. 4f, mean_log ${ }_{2} \mathrm{PDR}_{\mathrm{CA} \_ \text {lethal EC50 }}=-0.36$, mean_log $\log _{2} \mathrm{PDR}_{\mathrm{CA} \_ \text {total_EC50 }}=-0.20$, mean_log $2 \mathrm{PDR}_{\mathrm{IA}}$ lethal_EC50 $=0.94$, mean_log $2 \mathrm{PDR}_{\mathrm{CA} \_ \text {total_EC50 }}=1.17$ ).

The PDR distributions according to exposure times are shown in Fig. 4g and h. Results shown in Fig. 4h indicate that the smallest PDRs were achieved with the CA model and an exposure period of 72 hpe (mean_log ${ }_{2}$ PDR $\mathrm{CA}_{\text {CA2hpe_EC50 }}=-0.09$ ).

From these results we can conclude that mixture toxicity in ZFE, in general, was better predicted with the CA model with a range of $-1.32<\log _{2} \mathrm{PDR}<0.41$. Overall, the highest model accuracy in terms of smallest PDRs were found with the CA model for high effect concentrations (mean_log ${ }_{2} \mathrm{PDR}_{\mathrm{CA} \_ \text {EC90 }}=-0.15$ ) and the $72 \mathrm{~h}$ exposure period (mean_log ${ }_{2} \mathrm{PDR}_{\mathrm{CA} \_72 \mathrm{hpe}}=-0.09$ ). However, for the IA model, the highest model accuracy was achieved when low mixture effects obtained after short exposure durations were estimated (mean_log $\log _{2} \mathrm{PDR}_{\mathrm{IA}}$ ${ }_{\mathrm{EC} 10}=0.941$, mean_log $\left.\log _{2} \mathrm{PDR}_{\mathrm{IA} \_24 \mathrm{hpe}}=0.978\right)$. The considered phenotype did not seem to influence the prediction quality significantly.

\section{Mixture toxicity is dependent on age of ZFE at exposure start and on exposure duration}

Next to concentrations of chemicals or mixtures, toxicities are also dependent on the duration of exposure. In a developing system such as the ZFE, the time point of exposure, i.e., the age of the embryo at exposure start, is an additional determining factor for toxicity. An overview of time dependent mortality for all analyzed single substances and mixtures in this study is given in Table 4. ZFE were exposed at an early $(0 \mathrm{hpf})$ and a later time point (24 hpf) and effects were determined every $24 \mathrm{~h}$ during development. We calculated a factor for time dependence
Table 4 Time dependence (td) of single substance and mixture toxicity

\begin{tabular}{|c|c|c|}
\hline Substance & td_lethal_early & td_lethal_late \\
\hline Benzo[a]pyrene & * & * \\
\hline Benzo[b]fluoranthene & $*$ & * \\
\hline Bisphenol A & 0.658 & 0.680 \\
\hline Carbendazim & 0.922 & 0.431 \\
\hline Chlorophene & 0.402 & 0.473 \\
\hline Cyprodinil & 1.102 & 0.422 \\
\hline Diazinon & 1.124 & 0.866 \\
\hline Diclofenac & 0.053 & 0.396 \\
\hline Diuron & 1.045 & * \\
\hline Genistein & 0.606 & 0.018 \\
\hline Naphthalene & 1.126 & 0.554 \\
\hline Naproxen & 0.604 & 0.241 \\
\hline Propiconazole & 0.855 & 0.819 \\
\hline Triclosan & 0.371 & 0.520 \\
\hline Triphenyl phosphate & 0.755 & 0.982 \\
\hline Mean_single substances & 0.740 & 0.534 \\
\hline $\operatorname{mixA}$ & - & 0.223 \\
\hline $\operatorname{mixB} .1$ & 0.978 & - \\
\hline $\operatorname{mixB} .2$ & - & 0.713 \\
\hline $\operatorname{mixC} .1$ & 0.848 & - \\
\hline $\operatorname{mixC} .2$ & - & 0.525 \\
\hline $\operatorname{mixD}$ & - & - \\
\hline mixE.1 & 0.330 & 0.556 \\
\hline mixE.2 & 0.636 & 0.362 \\
\hline $\operatorname{mixF}$ & - & 0.712 \\
\hline mean_mixtures & 0.698 & 0.561 \\
\hline
\end{tabular}

Shown is the time dependence (td) of toxicity for lethal effects obtained after starting exposures early $(0 \mathrm{hpf})$ and late $(24 \mathrm{hpf})$, respectively

$\mathrm{Td}$ is the ratio $\mathrm{LC}_{50}$ (longest exposure duration) / $\mathrm{LC}_{50}$ (shortest exposure duration)

* No CRC at tested concentrations, - no toxicity tested

(td) of toxicity by calculating the ratio between the $\mathrm{LC}_{50}$ obtained after the longest exposure duration and $\mathrm{LC}_{50}$ obtained after the shortest exposure duration. For the single substance exposures, we observed four possible cases of time dependent toxicity: (1) no time dependence at all (9/25 cases, e.g., diuron), (2) similar time dependence for early and late exposures (3/12, e.g., bisphenol A), (3) time dependence is larger in early exposures (3/12 cases, e.g., diclofenac), and (4) time dependence is larger in late exposures (6/12 cases, e.g., genistein).

Single chemical or mixture toxicity was considered as time dependent when $\mathrm{td}$ was below 0.8 or greater than 1.2. Chemicals with a time dependent toxicity have a varying impact on the toxicity of a respective mixture over time, when the mixture ratio is kept constant. For instance, the toxic units of the chemicals in the mixtures change during the exposure duration and the impact of 
independently and similarly acting components in a mixture may vary over time (Additional file 2: Figure S22). In 16 out of 25 cases (68\%) time dependent toxicity was detected for single substances, whereas mixture toxicity was time dependent in almost all cases investigated therein $(8 / 10,(80 \%))$. Two exceptions were mixB.1 and mixC.1 which were directly exposed after hatching and only for $48 \mathrm{~h}$. On average we found that an early exposure start resulted in less time dependence in toxicity compared to exposures that started at later stages, i.e., with older ZFE (td_mean_early $=0.72$, td_mean_late $=0.55$ ). This means that the toxicity doubles during the course of longer exposure periods when ZFE are exposed in an advanced developmental stage.

\section{Increased steepness of mixture concentration-response curves}

In this study we found that all mixtures induce steep CRCs. We classified a CRC as steep when the slope (calculated as quotient of $\left.\mathrm{EC}_{75} / \mathrm{EC}_{50}\right)$ was $<2$ as an $\mathrm{EC}_{75} /$ $\mathrm{EC}_{50}$ ratio of 2 means that doubling the $\mathrm{EC}_{50}$ concentration leads to a double fractional effect, which is what one would expect under the law of mass action. The slope distributions of ss_CRCs and mix_CRCs obtained for lethal and total effects and the four different exposure periods (24, 48, 72, and $96 \mathrm{~h}$ ) are shown in Fig. 5 (purple: mix_CRC, pink: ss_CRC). The slopes of all analyzed mixtures were steep with a trend towards steeper CRC for longer exposure durations. Also, the slopes of ss CRCs were relatively steep but showed broader distributions. On average we found that a concentration increase of approximately $15 \%$ resulted in twice the effect in the zebrafish embryo toxicity test (see Table 5) based on the $\mathrm{LC} / \mathrm{EC}_{50}$. A detailed list of all data is presented in Addtional file 2: Tables S8-11.

\section{'Something from "nothing"' effects induced by mixtures in ZFE}

Mixture exposures can cause the induction of significant toxic effects even when mixture components are applied in concentrations that are not evoking toxicity individually. This phenomenon is also known as the principle 'something from "nothing"' $[17,18,22,24,36]$. Silva et al., for example, showed that a mixture of eight weak estrogenic compounds were able to evoke a significant combined effect in yeast although the mixture components were present below their individual NOEC [17]. In this study, we also detected a combined effect for all tested mixtures and were interested which concentrations of individual mixture components were necessary to evoke a significant mixture effect of $90 \%$ regarding either lethal but also sublethal and teratogenic endpoints $\left(\mathrm{EC}_{90}\right)$. The $\mathrm{EC}_{90}$ of a mixture was selected, because this

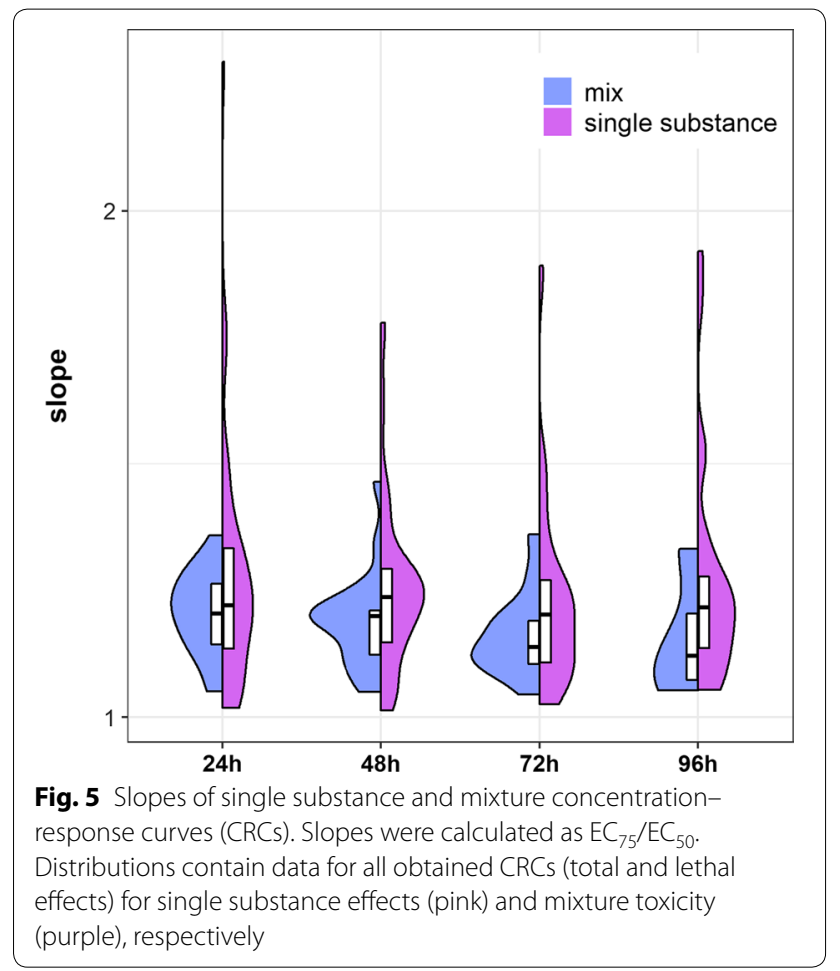

Table 5 Slope calculation for single substance (ss) and mixture (mix) concentration-response curves

\begin{tabular}{lllll}
\hline & Min. & Median & Mean & Max. \\
\hline ss & 1.01 & 1.17 & 1.21 & 2.45 \\
Ss_24hpe & 1.01 & 1.17 & 1.22 & 2.45 \\
Ss_48hpe & 1.01 & 1.18 & 1.21 & 1.72 \\
ss_72hpe & 1.02 & 1.15 & 1.18 & 1.86 \\
ss_96hpe & 1.04 & 1.16 & 1.22 & 1.89 \\
mix & 1.03 & 1.14 & 1.14 & 1.38 \\
mix_24hpe & 1.04 & 1.12 & 1.13 & 1.23 \\
mix_48hpe & 1.04 & 1.15 & 1.14 & 1.38 \\
mix_72hpe & 1.03 & 1.10 & 1.12 & 1.28 \\
mix_96hpe & 1.04 & 1.09 & 1.12 & 1.26 \\
\hline
\end{tabular}

value still implies a remarkable joint effect but also guarantees statistical robustness. The results are exemplarily shown for a rather simple (mixC.1) and more complex mixture (mixE.1) in Fig. 6. The grey bars depict the $\mathrm{EC}_{90}$ induced by the specific mixture concentration obtained after 24, 48, 72, and 96 hpe, respectively. The light blue (CA) and yellow bars (IA) indicate the effects predicted by the models for the respective mixture concentration. The pink ( $24 \mathrm{~h}$ ), purple $(48 \mathrm{~h})$, orange $(72 \mathrm{~h})$ and rosé bars $(96 \mathrm{~h})$ depict the effects evoked by mixture components when applied individually at the concentration present in the mixture. The data shown in Fig. 6 indicate that a 

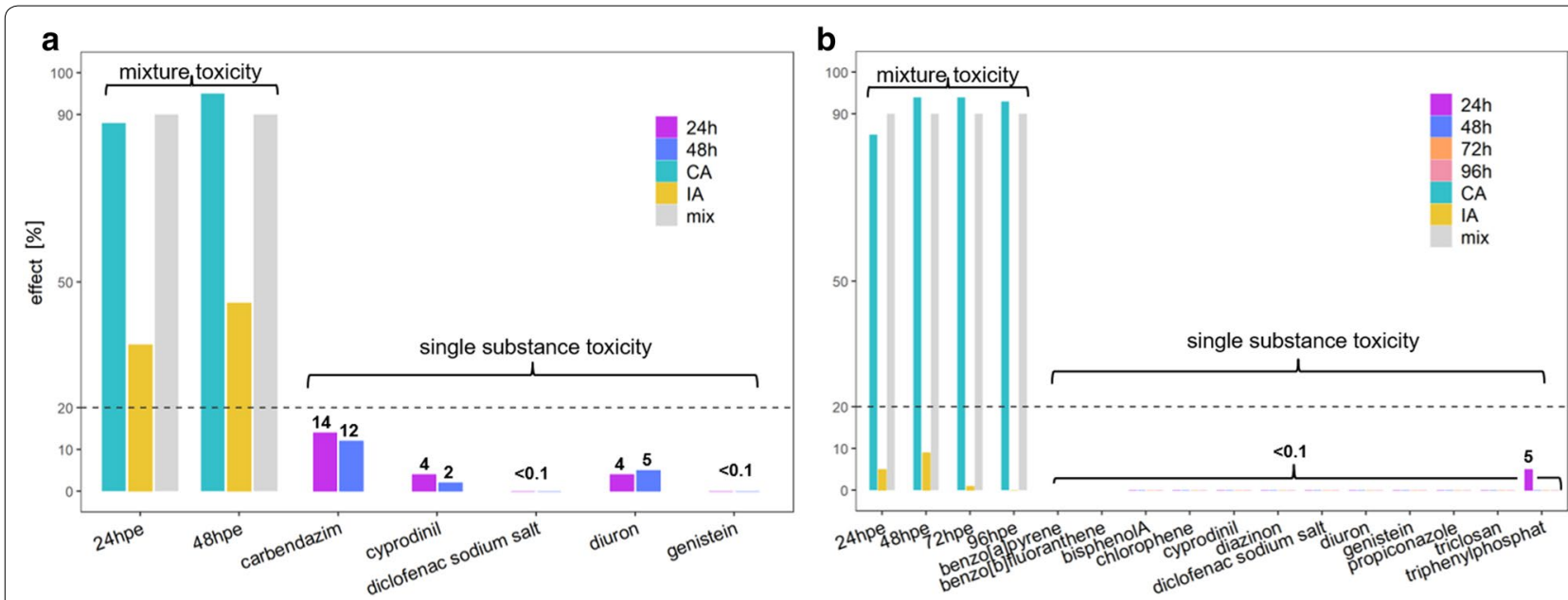

Fig. 6 'Something from "nothing"': Predicted effects modeled with CA (light blue) and IA (yellow) at mixture concentrations evoking 90\% effect in measurements (grey bars) and the effects evoked by single substances when applied individually at the concentrations present in the mixture at 24 hpe (pink), 48 hpe (purple), 72 hpe (orange) and 96 hpe (rosé). a mixC.1_lethal (LC $50 \_24 h=18.17 \mu \mathrm{M}, \mathrm{LC}$ 50_48h $\left.=21.42 \mu \mathrm{M}\right), \mathbf{b}$ mixE.1_total $\left(E_{90 \_24 h}=15.72 \mu \mathrm{M}, E_{90 \_48 h}=14.05 \mu \mathrm{M}, E_{90 \_72 h}=10.90 \mu \mathrm{M}, \mathrm{EC}_{90 \_96 h}=9.71 \mu \mathrm{M}\right)$

significant mixture effect was induced even though individual chemicals were applied in concentrations below their individual effect thresholds $\left(\mathrm{EC}_{20}\right.$, see "Methods" section). This phenomenon holds true for both mixtures but is even more distinct for the more complex, 12-compound mixture.

Surveying the entirety of results revealed that for a remarkable number of $90 \%$ (53 out of 59 cases) a combined effect of $90 \%$ is caused by a mixture constituted of very low concentrated chemicals (Additional file 2: Figure S11-21).

\section{Discussion}

For a reliable comparison of predicted and measured mixture toxicity, a valid and free-of-bias determination of toxicity values is necessary. Single substance and mixture toxicity observations, therefore, need to be performed in a comparable manner and a high-quality single substance toxicity determination is the major requirement to trustfully predict mixture toxicity. Thus, toxicity determination of single substances as well as of mixtures was performed until an appropriately resolved concentration-response curve (CRC) was obtained. To minimize the influence of experimental errors and batch effects, we analyzed three technical replicates per tested concentration and further performed multiple independent experiments starting at different days (minimum two, maximum five). The individually obtained CRCs for mixture constituents were further used to predict mixture toxicity with the model of Concentration Addition (CA) and Independent Action (IA) for, respectively, designed mixtures. All mixtures were tested in the zebrafish embryo acute toxicity assay (ZFET) and obtained measurements were compared with respective predictions. Due to conceptual differences, CA and IA often provide different toxicity values. This is not necessarily the case but is, however, frequently observed when mixtures consist of multiple chemicals at low concentrations (e.g., [37]). In such cases, the predicted CRCs of both models span a concentration-effect space, the prediction window. The mixture ratio and applied chemical concentrations can influence the separation of the CA and IA model, hence the size of the prediction window. Whether or not both models need to be sufficiently distinguishable is dependent on the research question. For instance, if one is only interested in the detectability of a combined effect evoked by a mixture, it is neglectable that both models distinguish from each other whereas investigations about which model better describes the toxicity of a specific mixture require a clear separation of both models. In this study, we were, on the one hand, interested in whether or not mixture toxicity of a mixture consisting of only similarly or dissimilarly acting components is trustfully estimated by the appropriate model and, on the other hand, whether certain experimental factors, such as phenotype, exposure duration, and mixture potency, influence the prediction accuracy of the CA and IA model. For this, a clear separation of both models is a fundamental requirement. In this study, the distance of two predicted ECx values $(x=10,50,90)$ ranged from 1.4 to 5.5 with an average distance of 2.7 , hence CA and IA were sufficiently different. Meeting the requirements of high-quality and consistent toxicity observation as well as distinctive separation of toxicity predictions allowed 
us to reliably compare measured and predicted mixture toxicity values and evaluate the respective comparisons.

\section{Independent action might not be detectable with complex organisms and integrated endpoints}

Initially, we investigated the predictability of the toxicity of two specifically designed mixtures. One mixture contained only similarly acting substances (mixB), hence mixture toxicity expected to be predictable with the CA model, and another mixture contained only suspected dissimilarly acting constituents $(\mathrm{mixC})$, hence the IA model was assumed to best predict mixture toxicity. The measured mixture toxicity was reflected by CA in 6 out of 8 analyzed exposure scenarios for mixB, i.e., the obtained results met our hypothesis. In contrast, toxicity of mixC was not reflected by IA as expected (0 out of 8 cases) but was always located within the prediction window. It seems that in a complex organism, such as the ZFE, combined toxicity of similarly acting components is predictable with CA, whereas IA cannot reliably estimate the toxicity of a mixture consisting of dissimilarly acting components. Other studies in fish revealed similar findings. The combined effect of similarly acting components was predictable by CA in guppy [24] and fathead minnow [25]. A more controversial picture exists regarding the predictability of dissimilarly acting components with IA as effects were either not detectable against expectations [38] or were detected although multiple mixture components were suspected to act similarly by inducing narcosis [39]. Extending the literature research to other organisms revealed different results. In Gammarus pulex [40], mixture toxicity was underestimated by both models, whereas the combined effect of either similarly or dissimilarly acting components was proven to be predictable by the appropriate model in freshwater algae Scenedesmus vacuolatus (CA: [19, 20, 41], IA: [13]), luminescent bacteria Vibrio fischeri (CA: [21], IA: [12]) and Daphnia magna (CA: [22, 41]). It seems that the complexity of the organism determines whether or not the respective model is capable of predicting the combined effect of a mixture with the associated character.

Recalling the main principle of the IA model at which a combined effect is induced through interaction of mixture components with independent target sites by different modes of action, leads to the hypothesis that an independent action of chemicals may not be exerted in complex organisms [16]. One potential explanation could be derived from the absence of specific target sites in the investigated organism. For instance, the herbicide diuron, as part of the five component mixture with potentially dissimilarly acting substances, interferes with the photosystem II of plants and photosynthetic microorganisms [42]. In case an organism is lacking a functional photosystem, diuron is expected to not target a specific site and, therefore, is not acting via its specific mode of action (MoA) in zebrafish.

Complex interactions of chemicals with metabolic and signaling pathways could be another explanation for mismatches with the IA model. For instance, the phytoestrogen genistein, another suspected specifically and dissimilarly acting mixture component, has been shown to interact with estrogen receptors resulting in endocrine disruption. However, genistein was also identified to interfere with the aryl hydrocarbon receptor (AhR), e.g., as agonist in HepG2 cells [43] and rat liver [44], resulting in the induction of monooxygenases of the CYP1 family. The CYP1A1 and A2 enzymes play a crucial role in biotransformation of a diversity of chemicals and were shown to biotransform diuron to 3,4-dichloronaniline (3,4-DCA) [45] and genistein to dihydrogenistein and $2^{\prime}, 4^{\prime}, 6^{\prime}, 4^{\prime \prime}$-tetrahydroxy- $\alpha$ methyl-deoxybenzoin [46]. 3,4-DCA is suspected to influence anti-androgenic pathways which could further be related to endocrine disruption [45]. The parent compound diuron might, therefore, also be categorized into the same mode of action group as genistein. Gene expression analysis in ZFE also revealed an induction of CYP1 enzymes upon exposure to diuron [32]. Another component of mixC, diclofenac is known to be metabolized to $4{ }^{\prime}$-hydroxydiclofenac by CYP2C9 [47]. In gene expression studies with ZFE, diclofenac was found to induce cyp $2 k 19$ and $c y p 2 c 9$ [32, 48]. These are just examples for the induction of the biotransformation system and the list of metabolites could easily be extended.

The induction of biotransformation could lead to an increase of compound degradation of applied chemicals and the hereinafter following loss of specific action. However, the loss of specific action does not necessarily result in the total inactivation of chemicals. The resulting transformation products could still act via a similar and/or different MoA. For instance, metabolites could intercalate into the membrane, thus inducing unspecific narcotic effects. Cyprodinil, another component of mixC, was the only assumed baseline toxicant in this mixture. The biological activity of the different compounds might, therefore, change towards similar pathways such as narcosis. The results of our study and the considerations above indicate that independent action of chemicals in a mixture seems to be more likely for short exposure durations and low effect concentrations. It seems that independent MoAs of mixture components converge with increasing effective concentrations 
and longer exposure durations into joint unspecific pathways of disturbance in a complex organism.

\section{Mixture toxicity in ZFE can be predicted with the CA concept}

CRCs for experimentally determined mixture toxicities were located within the prediction window in 51 out of 59 cases (86\%) in this study. In 8 out of 59 cases (14\%), mixtures were even more toxic than predicted. Other studies performed with luminescent bacteria [12], algae [19], or ZFE [49] described similar results but their analyses were restricted to one selected mixture and specific exposure scenarios. Here, we demonstrate for the first time that mixture toxicity can be robustly estimated with the prediction window for a diversity of mixtures and exposure scenarios. In cases where the observed mixture toxicity was underestimated by both models, the deviations were not larger than one order of magnitude, respectively. Hence, the prediction window serves as a reliable tool to predict the concentration-effect space for mixture toxicity in ZFE.

Overall, the CA model predictions maximally deviated from measurements by a factor of $2.5(1.32<\log$ $\left.{ }_{2} \mathrm{PDR}<0.41\right)$. Regarding usually standard errors of ecotoxicological studies and concentration response determinations, this could be seen as reasonable prediction accuracy. The highest prediction accuracies with CA were achieved for higher effect concentrations and long exposure durations (e.g., mean_log ${ }_{2} \mathrm{PDR}_{\mathrm{CA} \_72 \mathrm{hpe}}$ $\mathrm{EC} 50=-0.09)$. The IA model revealed its highest model accuracy for low effect concentrations and shortterm exposures (mean_log ${ }_{2} \mathrm{PDR}_{\mathrm{IA} E \mathrm{EC} 10}=0.941$, mean $\log _{2} \mathrm{PDR}_{\mathrm{IA} \_24 \mathrm{hpe}}=0.978$ ), but was on average always less accurate in comparison to the CA model.

Predictions appeared to be slightly more robust when the totality of effects were inspected (lethal, sublethal, and teratogenic) but the inspected effect type seems to be less relevant concerning the performance of both models, in general. One possible explanation could be that both, lethal and total effects, are integrated effects, but the quantity of integrated endpoints and consequently the robustness of results increases when all recorded endpoints are merged and analyzed together.

While it remains to proofed whether or not mixture components can cause toxicity independently in complex model organisms, our results demonstrate, that the CA model was extremely robust and adequately estimated the toxicity of a large variety of mixtures and exposure scenarios within described margins of errors. Although the highest mixture toxicity prediction accuracies were achieved for longer exposures with high concentrations, CA-based predictions deviated from measurements by a factor of 2.5 only, even for mixtures with only suspected dissimilarly acting components and short-term exposures with low concentrations. Hence, the CA model, potentially applied with a safety factor, should provide a solid tool to predict mixture toxicity for environmental exposure scenarios, water quality and risk assessment. We, therefore, support the general request of ecotoxicologists to apply this model in water quality and environmental risk assessment $[7,50]$.

In conclusion, while the prediction window serves as a reliable tool to describe the concentration-effect space in which the toxicity of a mixture is to be expected, the CA model serves as robust and reliable tool to predict mixture toxicity, even for heterogeneous mixtures and a variety of exposure scenarios, and should, therefore, be considered for water quality and environmental risk assessment. The precise prediction quality of the CA and IA model is, however, dependent on specific experimental factors and should be considered when investigating mixture modes of action, potential interactions of the mixture constituent's pathways, and for detailed quantifications of combined effects [51].

\section{ZFE age and exposure durations determine mixture toxicity}

Toxicity in the ZFE is not only influenced by biotransformation processes but also by compound uptake kinetics and the developmental stage of the embryo. Our results show that longer exposure periods led to higher toxicity values in the majority of cases. This was even more apparent when ZFE were exposed at a subsequent developmental stage (at the age of $24 \mathrm{hpf}$ ). The increase in toxicity over time and a more pronounced time dependency of toxicity in older embryonic stages can be explained with toxicokinetic (e.g., chemical uptake kinetics and metabolism) and toxicodynamic (e.g., presence of certain target sites and functionality of pathways) processes. The developmental stage of the embryo determines the presence and function of molecular target sites, tissues, and organs. For instance, the liver, which is important to metabolize exogenous chemicals, starts budding in ZFE at the age of $24 \mathrm{hpf}$. It ends with the connection of liver bud and intestine at $50 \mathrm{hpf}$. Subsequently, the growth period begins and the liver undergoes remarkable changes in size and shape $[52,53]$. A functional liver is required for the biotransformation of many chemicals which can lead to either inactivation or detoxification, or bioactivation due to the production of toxic metabolites [54]. Evidence for bioactivation or detoxification can be derived from toxicity observations only when different exposure scenarios and time points are considered. While we found both, increased and decreased toxicity over time, for the single compounds, mixture toxicity increased over time in all cases. Furthermore, we found 
the mixture CRCs on average to be steeper than the single compound CRCs with an increased steepness over time. On average we found that a concentration increase of approximately $15 \%$ resulted in double the effect. It seems that a certain threshold concentration is needed to induce an adverse effect in a complex organism such as the ZFE. The convergence of metabolic, stress response, and/or individual signaling pathways at a certain point and/or the additive burden within such pathways due to the existence of different chemicals at the same time might explain these observations.

\section{Mixture exposure leads to the 'something from "nothing"' phenomenon}

In the environment, chemicals occur in multitudes of low concentrated mixtures rather than as individual entities. Although acute toxicity disappears more and more off the monitor of concern, the environmental status of European surface waters is still in very poor condition [55]. The potential interaction of low concentrated chemicals and their ability to evoke combined toxic effects has been intensely discussed to be a plausible explanation. During the last three decades, scientific evidence has verified the hypothesis that combined effects of chemicals may be induced even when all mixture constituents are applied in very low concentrations. Several studies, conducted in yeast [17], algae [13, 37], daphnids [22], gammarus [40, 56], and fathead minnow [18], reported the detection of combined effects which were always higher than the effects induced by mixture constituents if applied individually. It has been reported that combined toxic effects were even observable when chemicals were applied in concentrations below their individual effect thresholds. This 'something from "nothing"' phenomenon could be shown for similarly acting components inducing narcosis $[22,24,36]$ as well as for independently acting substances [13] and a blend of both [14]. All these examples refer to situations, where experiments were specifically designed to investigate the impact of low concentrated chemicals and to assess their ability to evoke a combined effect. Additionally, the toxicity of the tested mixtures was usually elucidated for specific exposure scenarios. In this study, we demonstrate that 'something from "nothing"'effects could be detected although the mixtures were not designed to show this on purpose. Furthermore, we show that the detection of this phenomenon is not dependent on the examined exposure scenario and considered phenotype. However, exceptions derived from mixtures consisting of only a small number of chemicals (e.g., mixA containing only three chemicals) or short exposure periods, whereas the overall significance of the phenomenon 'something from "nothing" increased the more substances a mixture contained and the longer the ZFE were exposed to the mixture. Combining these findings with the chemical status quo of water bodies, at which water samples have been described to consist of 400-500 chemicals at low concentrations [3], is leading to the request of finally integrating mixture toxicity assessment into water quality assessment.

\section{Conclusions}

In this study, we show that the CA model provides a robust tool to predict mixture toxicity in zebrafish embryo. This conclusion is based on the investigation of 9 different mixtures and 31 different exposure scenarios and their toxicities in zebrafish embryos. The CA model correctly estimated the toxicity in $100 \%$ of analyzed mixtures when allowing a prediction deviation ratio of 2.5. This was not only the case for mixtures containing exclusively similarly acting components but was also true for mixtures that contained only dissimilarly acting components or a blend of both. We found experimentally determined mixture toxicity within the prediction window, the concentration-effect space that is spanned between CA and IA predictions, in 51 of 59 cases. In 8 of 59 cases mixture toxicity was underestimated with both models, whereas an overestimation was never observed. All investigated mixtures induced steep concentrationresponse curves and increased toxicity over time. Finally, remarkable joint toxic effects were detectable for the majority of analyzed mixtures even if mixture constituents were present in concentrations that failed to evoke individual compound toxicity. This study demonstrates that combined effects of chemicals need to be considered as potential risk and that the CA model can serve as a robust tool to estimate mixture toxicity.

\section{Supplementary information}

Supplementary information accompanies this paper at https://doi. org/10.1186/s12302-020-00409-3.

Additional file 1: TableS12_chemical_information.xlsx.

Additional file 2: Supplementary_information.pdf.

Additional file 3:TableS13_toxicity_raw_data.xlsx.

Abbreviations

CA: Concentration Addition; CRC: Concentration-response curve; EC: Effect concentration (concentration that induces a certain effect in $\mathrm{x} \%$ of the organisms); hpf: Hours post fertilization; IA: Independent Action; LC: lethal concentration (concentration that induces lethality in $\mathrm{x} \%$ of the organisms); mix_CRC: Mixture concentration-response curve; MoA: Mode of action; NOEC: No observed effect concentration; ss_CRC: Single substance concentrationresponse curve; ZFE: Zebrafish embryo; ZFET: Zebrafish embryo acute toxicity assay; COX: Cyclooxygenase; AhR: Aryl hydrocarbon receptor; CAS RN: Chemical Abstracts Service Registry Number; td:Time dependence.

\section{Acknowledgements}

We gratefully acknowledge Nicole Schweiger for zebrafish embryo maintenance and thank David Leuthold, Madeleine Ammar, Silke Aulhorn, Daniela 
Taraba, Johanna Knapp, Jelena Fix, Jona Schulze, and Janna Kuhlmann for providing toxicity data of single substances and mixtures. Additionally, we would also like to thank Martin Scholze for providing an excel sheet for mixture toxicity prediction.

\section{Authors' contributions}

Conceptualization: GJ, RA, and WB. Investigations: GJ and JK. Data fitting/ modelling: GJ and AS. Data curation: GJ and JK. Data analysis: GJ. Visualization: GJ. Project administration: WB. Funding acquisition: RA and WB. Writing: GJ and WB. Review and editing: all. All authors read and approved the final manuscript.

\section{Funding}

Open Access funding enabled and organized by Projekt DEAL. This work was funded by the European Union 7th Framework Programme project SOLUTIONS (Grant Agreement No. 603437) and supported by the UFZ research unit CITE (Chemicals In The Environment).

\section{Availability of data and materials}

Supplementary figures and tables (Additional files 1, 2, 3)

\section{Ethics approval and consent to participate}

Not applicable.

\section{Consent for publication}

Not applicable.

\section{Competing interests}

The authors declare that they have no competing interests.

\section{Author details}

${ }^{1}$ Department of Bioanalytical Ecotoxicology, UFZ-Helmholtz Centre for Environmental Research, Permoserstr. 15, 04318 Leipzig, Germany. ${ }^{2}$ Institute for Environmental Research, RWTH Aachen University, Worringerweg 1, 52074 Aachen, Germany.

Received: 15 June 2020 Accepted: 18 September 2020

Published online: 26 October 2020

\section{References}

1. Report on the Environment - Chemicals Used on Land. https://www.epa. gov/report-environment/chemicals-used-land

2. Schwarzenbach RP (2006) The challenge of micropollutants in aquatic systems. Science 313:1072-1077. https://doi.org/10.1126/science.11272 91

3. Busch W, Schmidt S, Kühne R et al (2016) Micropollutants in European rivers: a mode of action survey to support the development of effect-based tools for water monitoring. Environ Toxicol Chem 35:1887-1899. https:// doi.org/10.1002/etc.3460

4. Calamari D, Vighi M (1992) A proposal to define quality objectives for aquatic life for mixtures of chemical substances. Chemosphere 25:531-542. https://doi.org/10.1016/0045-6535(92)90285-Y

5. de Zwart D, Posthuma L (2005) Complex Mixture Toxicity For Single and Multiple Species: proposed Methodologoies. Environ Toxicol Chem 24:2665. https://doi.org/10.1897/04-639R.1

6. Kienzler A, Bopp SK, van der Linden S et al (2016) Regulatory assessment of chemical mixtures: requirements, current approaches and future perspectives. Regul Toxicol Pharmacol 80:321-334. https://doi.org/10.1016/J. YRTPH.2016.05.020

7. Kortenkamp A, Faust M (2018) Regulate to reduce chemical mixture risk. Science 361:224-226

8. Escher BI, Stapleton HM, Schymanski EL (2020) Tracking complex mixtures of chemicals in our changing environment. Science 367:388-392. https://doi.org/10.1126/science.aay6636

9. Loewe S, Muischnek H (1926) Über Kombinationswirkungen1 Mitteilung: Hilfsmittel der Fragestellung. Naunyn-Schmiedebergs Arch Exp Pathol Pharmakol. 114:313-326
10. Kortenkamp A, Faust M, Scholze M, Backhaus T (2007) Low-level exposure to multiple chemicals: reason for human health concerns? Environ Health Perspect 115:106-114. https://doi.org/10.1289/ehp.9358

11. Bliss Cl (1939) The toxicity of poisons applied jointly. Ann Appl Biol 26:585-615

12. Backhaus T, Scholze M, Grimme LH (2000) The single substance and mixture toxicity of quinolones to the bioluminescent bacterium Vibrio fischeri. Aquat Toxicol 49:49-61. https://doi.org/10.1016/S0166 $-445 \times(99) 00069-7$

13. Faust M, Altenburger R, Backhaus T et al (2003) Joint algal toxicity of 16 dissimilarly acting chemicals is predictable by the concept of independent action. Aquat Toxicol 63:43-63. https://doi.org/10.1016/S0166 $-445 \times(02) 00133-9$

14. Altenburger R, Scholze M, Busch W et al (2018) Mixture effects in samples of multiple contaminants - an inter-laboratory study with manifold bioassays. Environ Int 114:95-106. https://doi.org/10.1016/J.ENVIN T.2018.02.013

15. Grimme LH, Altenburger R, Backhaus T, Bödeker W, Faust M, Scholze M (1998) Vorhersagbarkeit und Beurteilung der aquatischen Toxizität von Stoffgemischen: Multiple Kombinationen von unähnlich wirkenden Substanzen in niedrigen KonzentrationenNo Title. Leipzig

16. Boedeker W, Drescher K, Altenburger R et al (1993) Combined effects of toxicants: the need and soundness of assessment approaches in ecotoxicology. Sci Total Environ 134:931-939. https://doi.org/10.1016/S0048 -9697(05)80100-7

17. Silva E, Rajapakse N, Kortenkamp A (2002) Something from "nothing"-ight weak estrogenic chemicals combined at concentrations below NOECs produce significant mixture effects. Environ Sci Technol 36:1751-1756. https://doi.org/10.1021/es0101227

18. Thrupp TJ, Runnalls TJ, Scholze M et al (2018) The consequences of exposure to mixtures of chemicals: something from 'nothing' and 'a lot from a little' when fish are exposed to steroid hormones. Sci Total Environ 619-620:1482-1492. https://doi.org/10.1016/j.scitotenv.2017.11.081

19. Faust M, Altenburger R, Backhaus T et al (2001) Predicting the joint algal toxicity of multi-component s-triazine mixtures at low-effect concentrations of individual toxicants. Aquat Toxicol 56:13-32. https://doi. org/10.1016/S0166-445X(01)00187-4

20. Junghans M, Backhaus T, Faust M et al (2006) Application and validation of approaches for the predictive hazard assessment of realistic pesticide mixtures. Aquat Toxicol 76:93-110. https://doi.org/10.1016/j.aquat ox.2005.10.001

21. Altenburger R, Backhaus T, Boedeker W et al (2000) Predictability of the toxicity of multiple chemical mixtures to Vibrio fischeri: mixtures composed of similarly acting chemicals. Environ Toxicol Chem 19:2341-2347. https://doi.org/10.1002/etc.5620190926

22. Deneer JW, Sinnige TL, Seinen W, Hermens JLM (1988) The joint acute toxicity to Daphnia magna of industrial organic chemicals at low concentrations. Aquat Toxicol 12:33-38. https://doi.org/10.1016/0166445X(88)90017-3

23. Cleuvers M (2003) Aquatic ecotoxicity of pharmaceuticals including the assessment of combination effects. Toxicol Lett 142:185-194. https://doi. org/10.1016/50378-4274(03)00068-7

24. Könemann H (1981) Fish toxicity tests with mixtures of more than two chemicals: a proposal for a quantitative approach and experimental results. Toxicology 19:229-238. https://doi.org/10.1016/0300483X(81)90132-3

25. Broderius S, Kahl M (1985) Acute toxicity of organic chemical mixtures to the fathead minnow. Aquat Toxicol 6:307-322. https://doi. org/10.1016/0166-445X(85)90026-8

26. Backhaus T, Altenburger R, Boedeker W et al (2000) Predictability of the toxicity of a multiple mixture of dissimilarly acting chemicals to Vibrio fischeri. Environ Toxicol Chem 19:2348-2356. https://doi.org/10.1002/ etc.5620190927

27. Kimmel CB, Ballard WW, Kimmel SR et al (1995) Stages of embryonic development of the zebrafish. Dev Dyn 203:253-310. https://doi. org/10.1002/aja.1002030302

28. Escher Bl, Eggen RIL, Schreiber U et al (2002) Baseline toxicity (Narcosis) of organic chemicals determined by in vitro membrane potential measurements in energy-transducing membranes. Environ Sci Technol 36:1971-1979. https://doi.org/10.1021/es015844c 
29. Klüver N, Vogs C, Altenburger R et al (2016) Development of a general baseline toxicity QSAR model for the fish embryo acute toxicity test. Chemosphere 164:164-173. https://doi.org/10.1016/j.chemospher e.2016.08.079

30. Maeder V, Escher BI, Scheringer M, Hungerbühler K (2004) Toxic ratio as an indicator of the intrinsic toxicity in the assessment of persistent, bioaccumulative, and toxic chemicals. Environ Sci Technol 38:3659-3666. https ://doi.org/10.1021/es0351591

31. Verhaar HJM, van Leeuwen CJ, Hermens JLM (1992) Classifying environmental pollutants. Chemosphere 25:471-491. https://doi. org/10.1016/0045-6535(92)90280-5

32. Schüttler A, Altenburger R, Ammar M et al (2019) Map and model_-moving from observation to prediction in toxicogenomics. Gigascience. https ://doi.org/10.1093/gigascience/giz057

33. (2013) Test No. 236: Fish Embryo Acute Toxicity (FET) Test. OECD

34. Ritz C, Baty F, Streibig JC, Gerhard D (2015) Dose-response analysis using R. PLOS ONE 10:e0146021. https://doi.org/10.1371/journal.pone.0146021

35. Grimme LH, Altenburger R, Backhaus T, Boedeker W, Faust M, Scholze M (1998) Vorhersagbarkeit und Beurteilung von Mischungen

36. Hermens J, Canton H, Janssen P, De Jong R (1984) Quantitative structureactivity relationships and toxicity studies of mixtures of chemicals with anaesthetic potency: acute lethal and sublethal toxicity to Daphnia magna. Aquat Toxicol 5:143-154. https://doi.org/10.1016/0166445X(84)90005-5

37. Walter H, Consolaro F, Gramatica P et al (2002) Mixture toxicity of priority pollutants at no observed effect concentrations (NOECs). Ecotoxicology 11:299-310. https://doi.org/10.1023/A:1020592802989

38. Belden JB, Lydy MJ (2006) Joint toxicity of chlorpyrifos and esfenvalerate to fathead minnows and midge larvae. Environ Toxicol Chem 25:623-629. https://doi.org/10.1897/05-370R.1

39. Shao Y, Chen Z, Hollert H et al (2019) Toxicity of 10 organic micropollutants and their mixture: implications for aquatic risk assessment. Sci Total Environ 666:1273-1282. https://doi.org/10.1016/j.scitotenv.2019.02.047

40. Adam O, Badot P-M, Degiorgi F, Crini G (2009) Mixture toxicity assessment of wood preservative pesticides in the freshwater amphipod Gammarus pulex (L.). Ecotoxicol Environ Saf 72:441-449. https://doi.org/10.1016/j. ecoenv.2008.07.017

41. Cleuvers M (2004) Mixture toxicity of the anti-inflammatory drugs diclofenac, ibuprofen, naproxen, and acetylsalicylic acid. Ecotoxicol Environ Saf 59:309-315. https://doi.org/10.1016/S0147-6513(03)00141-6

42. Wessels JSC, van der Veen R (1956) The action of some derivatives of phenylurethan and of 3-phenyl-1,1-dimethylurea on the Hill reaction. Biochim Biophys Acta 19:548-549. https://doi.org/10.1016/00063002(56)90481-4

43. Zhang S, Qin C, Safe SH (2003) Flavonoids as aryl hydrocarbon receptor agonists/antagonists: effects of structure and cell context. Environ Health Perspect 111:1877-1882. https://doi.org/10.1289/ehp.6322

44. Perepechaeva ML, Grishanova YA (2012) In vivo effects of genistein, herbimycin a and geldanamycin on rat hepatic cytochrome P4501A. J Biophys Chem 03:334-340. https://doi.org/10.4236/jbpc.2012.34041

45. Zhou S (2018) Cytochrome P450 2D6, 1st edn. CRC Press, Boca Raton

46. Heinonen S-M, Hoikkala A, Wähälä K, Adlercreutz H (2003) Metabolism of the soy isoflavones daidzein, genistein and glycitein in human subjects.
J Steroid Biochem Mol Biol 87:285-299. https://doi.org/10.1016/j.jsbmb 2003.09.003

47. Zhou S-F, Zhou Z-W, Yang L-P, Cai J-P (2009) Substrates, Inducers, Inhibitors and Structure-Activity Relationships of Human Cytochrome P450 2C9 and Implications in Drug Development. Curr Med Chem 16:34803675. https://doi.org/10.2174/092986709789057635

48. Poon KL, Wang X, Lee SGP et al (2017) Transgenic Zebrafish reporter lines as alternative in vivo organ toxicity models. Toxicol Sci. https://doi. org/10.1093/toxsci/kfw250

49. Schmidt S, Busch W, Altenburger R, Küster E (2016) Mixture toxicity of water contaminants-effect analysis using the zebrafish embryo assay (Danio rerio). Chemosphere 152:503-512. https://doi.org/10.1016/j. chemosphere.2016.03.006

50. Vighi M, Altenburger R, Arrhenius Å et al (2003) Water quality objectives for mixtures of toxic chemicals: problems and perspectives. Ecotoxicol Environ Saf 54:139-150. https://doi.org/10.1016/S0147-6513(02)00047-7

51. Altenburger R, Schmitt H, Schüürmann G (2005) Algal toxicity of nitrobenzenes: combined effect analysis as a pharmacological probe for similar modes of interaction. Environ Toxicol Chem 24:324. https://doi. org/10.1897/04-032R.1

52. Field HA, Ober EA, Roeser T, Stainier DY (2003) Formation of the digestive system in zebrafish. I. liver morphogenesis. Dev Biol 253:279-290. https:// doi.org/10.1016/S0012-1606(02)00017-9

53. Tao T, Peng J (2009) Liver development in zebrafish (Danio rerio). J Genet Genomics 36:325-334. https://doi.org/10.1016/S1673-8527(08)60121-6

54. Kärki NT (1976) Mechanisms of toxicity and metabolism. Elsevier, Amsterdam

55. EEA Ecological status of surface water bodiesNo Title. In: 03.07.2018. https://www.eea.europa.eu/themes/water/european-waters/water-quali ty-and-water-assessment/water-assessments/ecological-status-of-surfa ce-water-bodies

56. Charles J, Crini G, Degiorgi F et al (2014) Unexpected toxic interactions in the freshwater amphipod Gammarus pulex (L.) exposed to binary copper and nickel mixtures. Environ Sci Pollut Res 21:1099-1111. https://doi. org/10.1007/s11356-013-1978-1

57. Arrhenius $\AA$, Grönvall F, Scholze M et al (2004) Predictability of the mixture toxicity of 12 similarly acting congeneric inhibitors of photosystem II in marine periphyton and epipsammon communities. Aquat Toxicol 68:351-367. https://doi.org/10.1016/j.aquatox.2004.04.002

\section{Publisher's Note}

Springer Nature remains neutral with regard to jurisdictional claims in published maps and institutional affiliations.

\section{Submit your manuscript to a SpringerOpen ${ }^{\circ}$ journal and benefit from:}

- Convenient online submission

- Rigorous peer review

- Open access: articles freely available online

- High visibility within the field

Retaining the copyright to your article

Submit your next manuscript at springeropen.com 